\title{
UDC 513.21
}

SCOPUS CODE 1701

https://doi.org/10.36073/1512-0996-2021-1-55-74

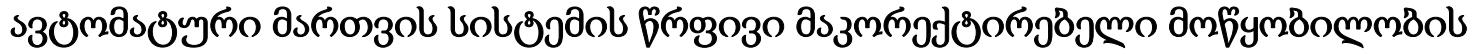 зsங

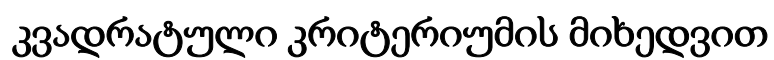

Bsçпо з3susmos

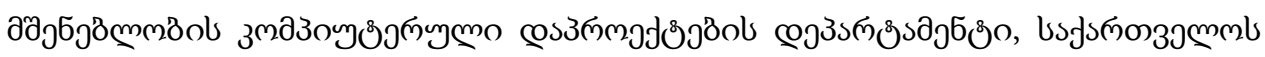

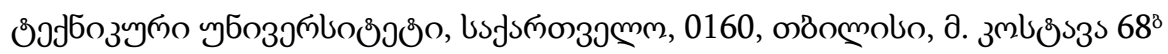

E-mail: b.gvasalia@gtu.ge

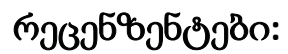

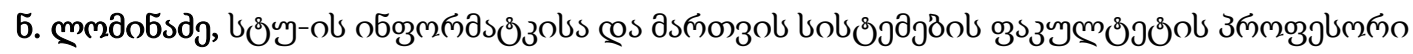

E-mail: n.lominadze@gtu.ge

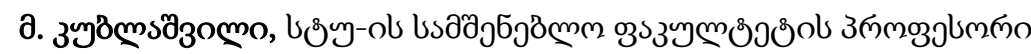

E-mail: m.kublashvili@gtu.ge

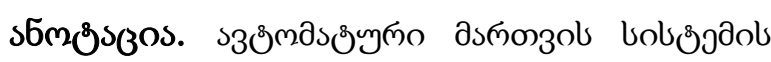

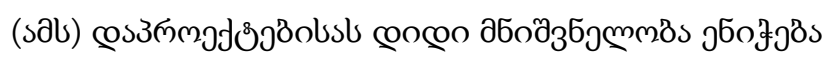

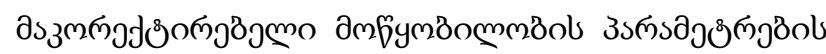

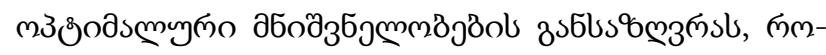

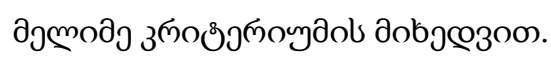

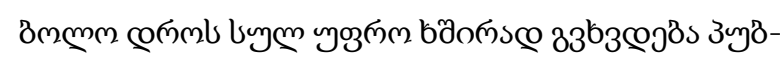

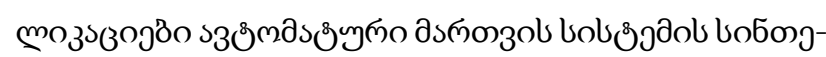
bol samzs

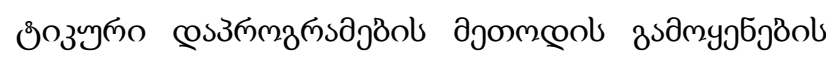
aglubga [1,2].

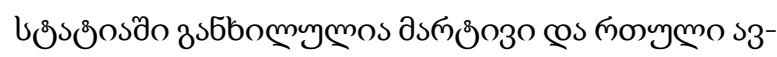

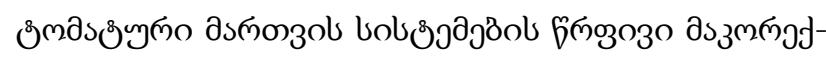

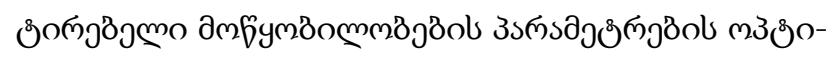

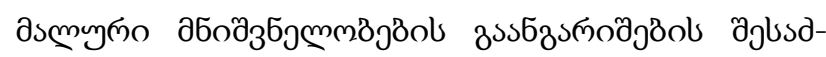
млдемпдјв̈о.

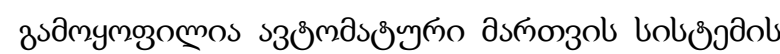

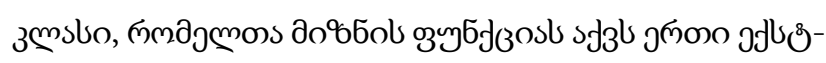

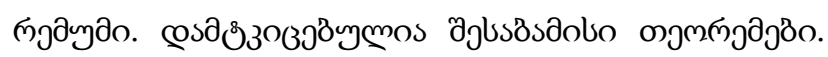

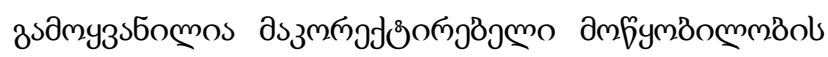

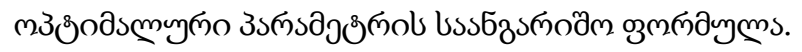

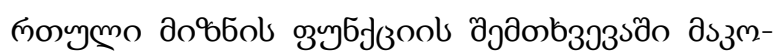

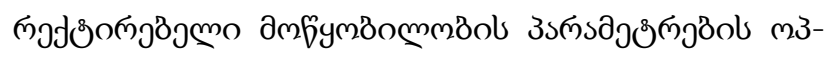

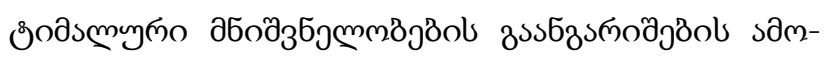

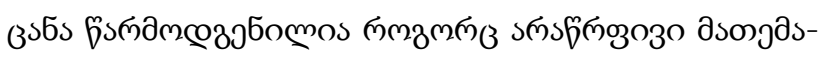

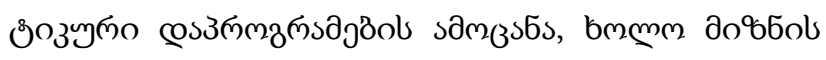

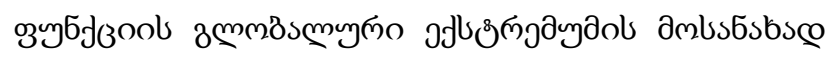

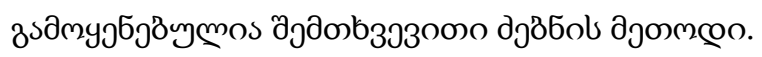

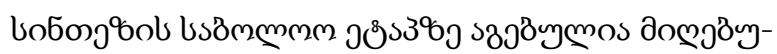

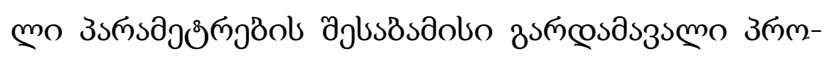

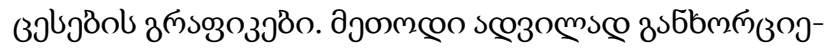

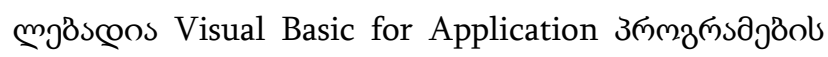

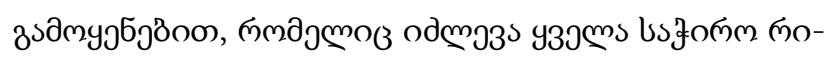

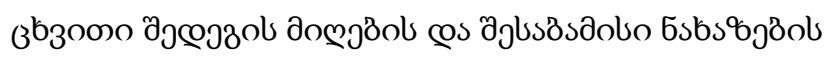

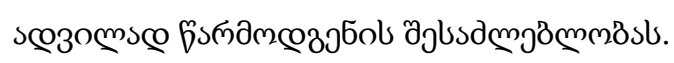




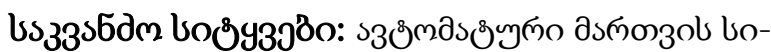

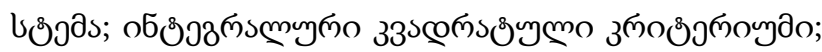

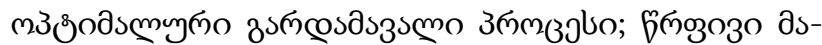

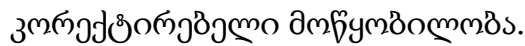

\section{gjuszsmo}

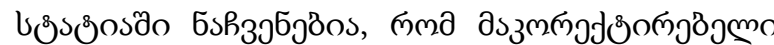

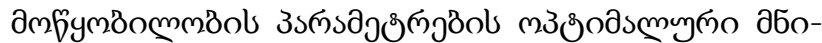

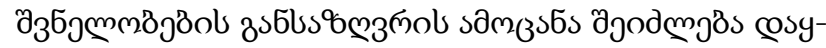

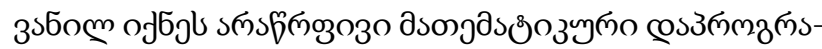

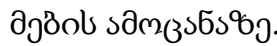

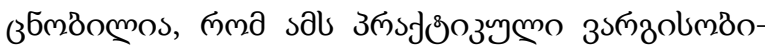

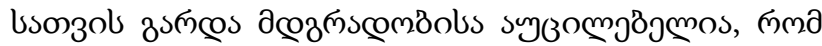

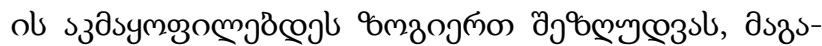

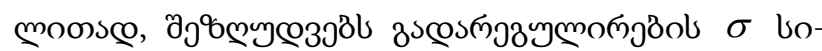

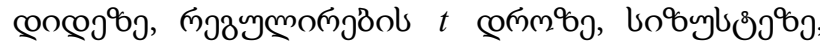

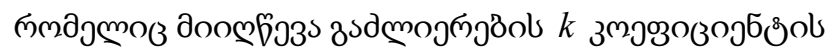

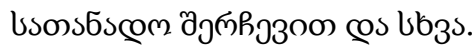

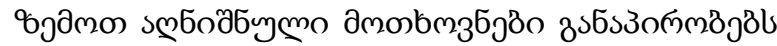

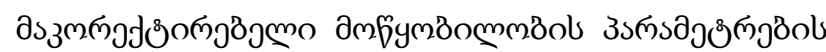

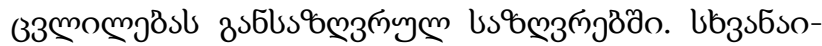

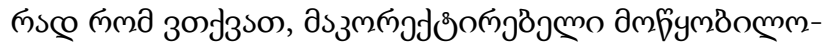

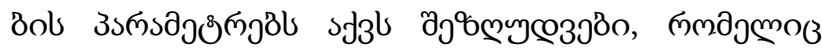

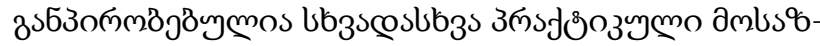

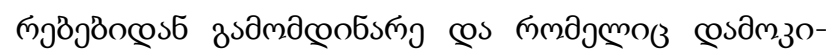

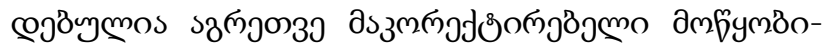

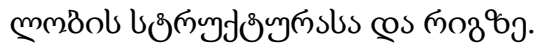

\section{domooscen Бsfiomo}

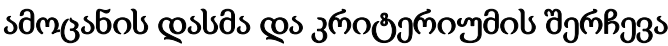

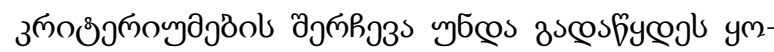

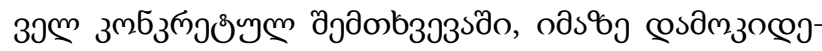

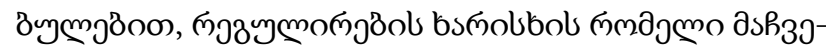

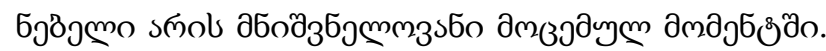

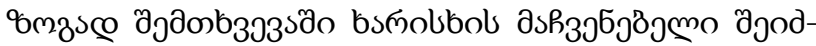

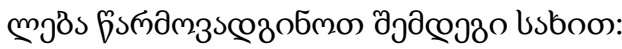

$$
I_{0}=I_{0}\left(x_{1}, x_{2}, \ldots, x_{n}\right),
$$

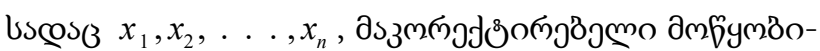

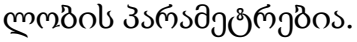

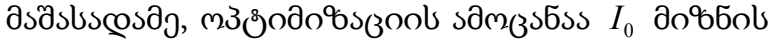

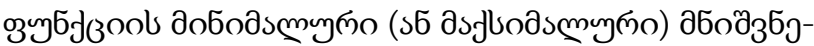

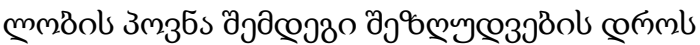

$$
g_{i}\left(x_{1}, x_{2}, \ldots, x_{n}\right) \geq 0, i=1,2, \ldots I_{g},
$$

cos

$$
h_{i}\left(x_{1}, x_{2}, \ldots, x_{n}\right)=0, i=1,2, \ldots I_{h},
$$

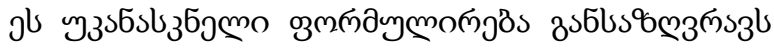

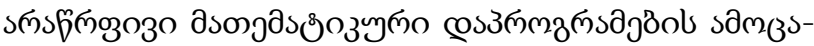

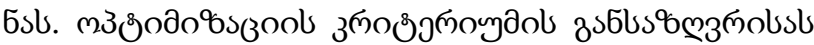

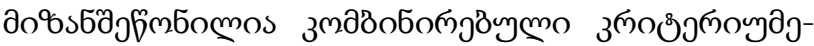

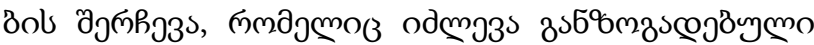

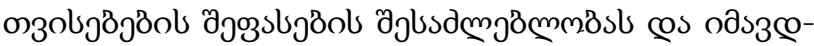

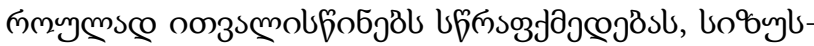

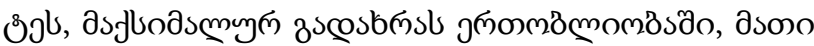

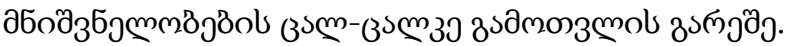

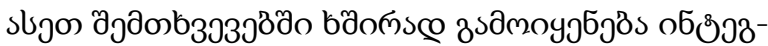

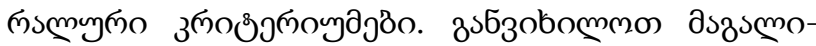

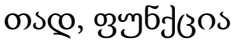

$$
I_{0}=\int_{0}^{\infty} y^{2}(t) d t
$$

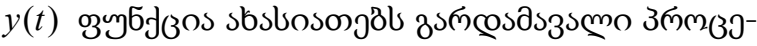

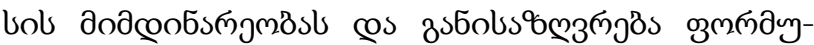

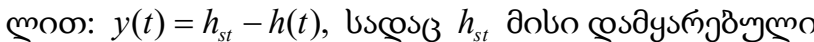

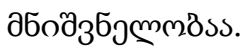

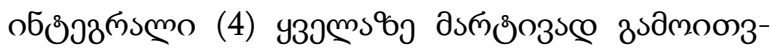

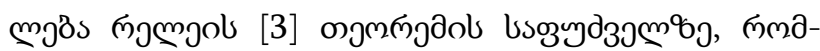

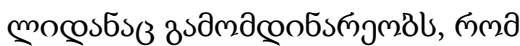




$$
I_{0}=\int_{0}^{\infty} y^{2}(t) d t=\frac{1}{\pi} \int_{0}^{\infty}\left|Y_{m, n}(j \omega)\right|^{2} d \omega,
$$

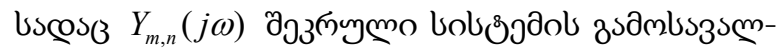

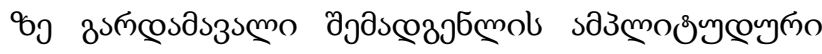

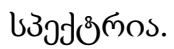

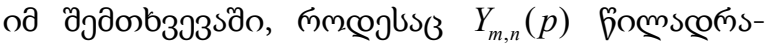

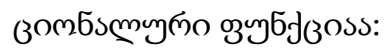

$$
\begin{gathered}
Y_{m, n}(p)=\frac{B(p)}{C(p)}= \\
=\frac{b_{m} p^{m}+b_{m-1} p^{m-1}+\ldots+b_{1} p+b_{0}}{c_{n} p^{n}+c_{n-1} p^{n-1}+\ldots .+c_{1} p+c_{0}} .
\end{gathered}
$$

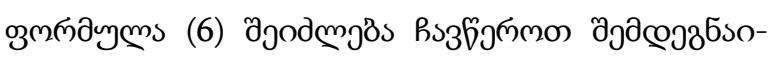
๓́sc:

$$
I_{0}=\frac{1}{\pi} \int_{0}^{\infty} \frac{B(j \omega) \cdot B(-j \omega)}{C(j \omega) \cdot C(-j \omega)} d \omega .
$$

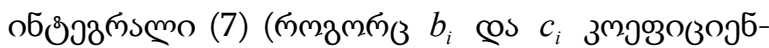

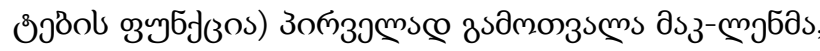

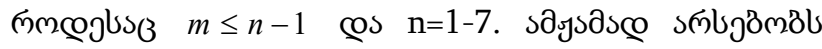

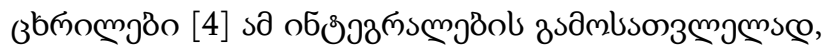

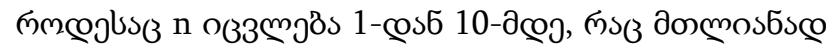

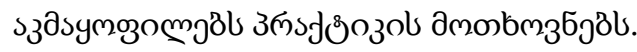

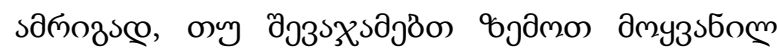
дmbs\%๓

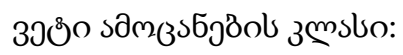

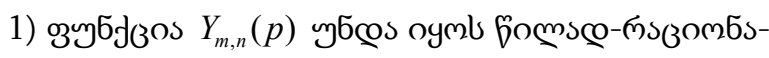

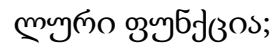

2) $Y_{m, n}(p)$ вйб

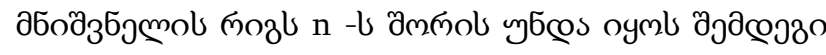

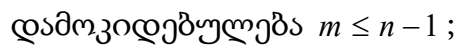

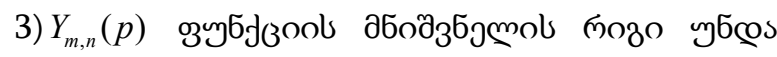

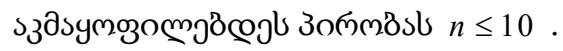

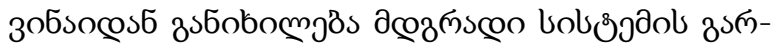

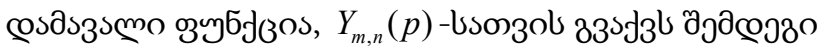

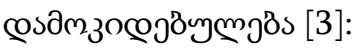

$$
Y_{m, n}(p)=\frac{W_{c l}(o)-W_{c l}(p)}{p},
$$

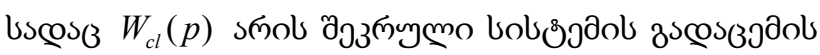

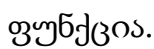

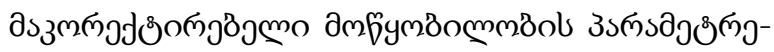

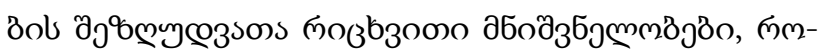

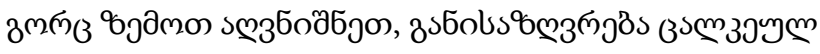

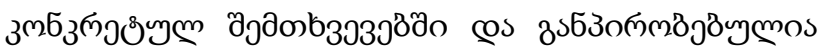

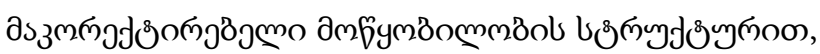

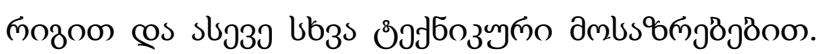

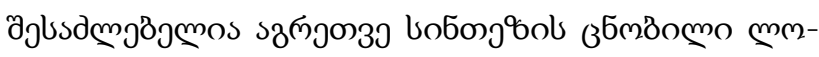

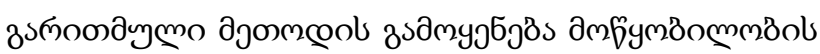

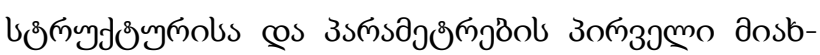

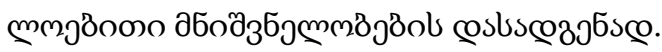

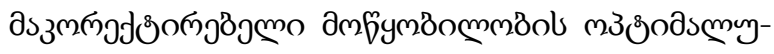

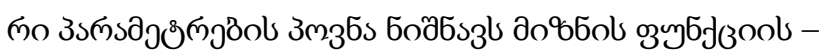

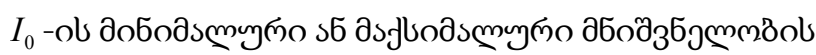

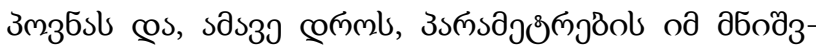

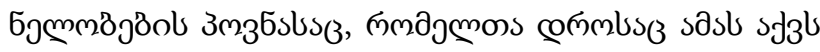
sқзомо.

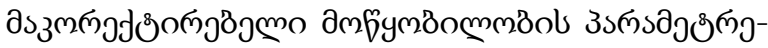

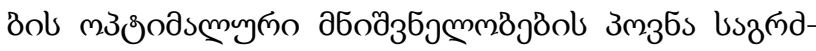

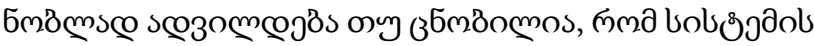

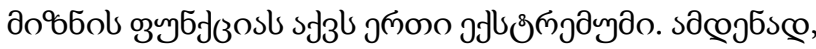

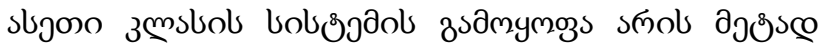

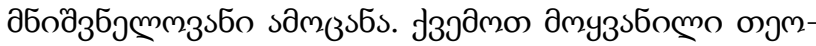

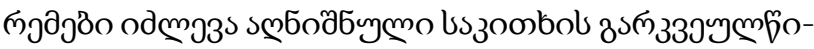

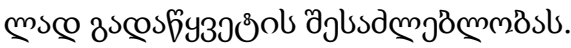




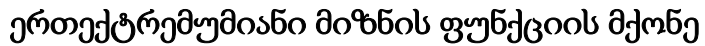

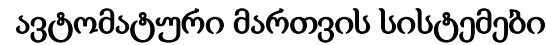

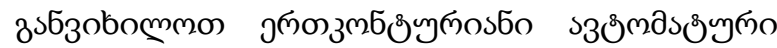

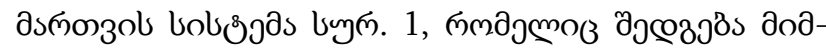

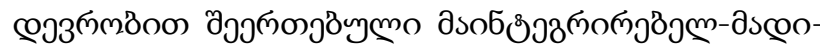

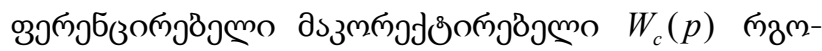

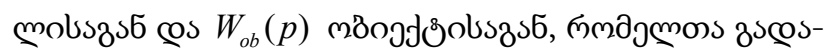

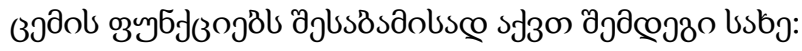

$$
W_{c}(p)=\frac{\tau p+1}{\alpha \tau \quad p+1},
$$

cos

$$
W_{o b}(p)=\frac{k}{p(T p+1)},
$$

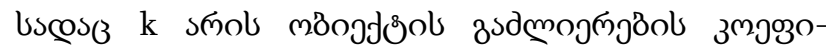

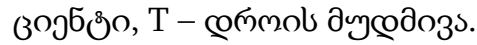

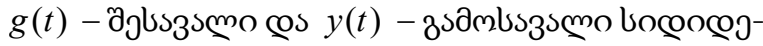

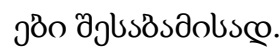

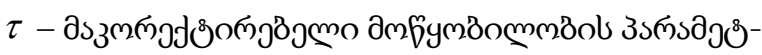
๓о.

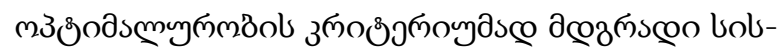

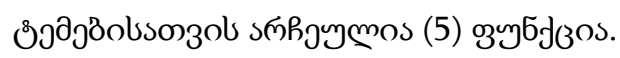

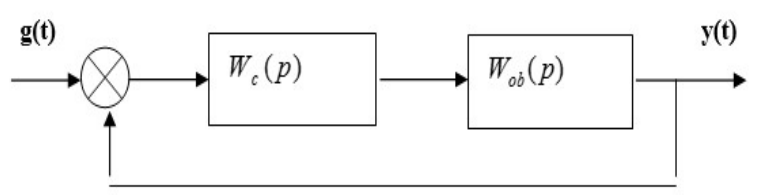

๒งyต. 1.

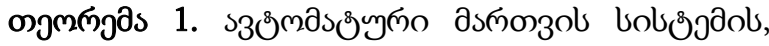

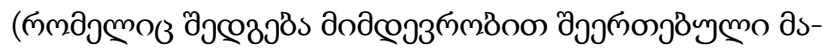

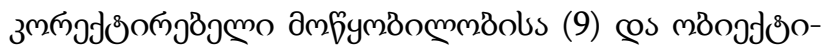
uszast (10), bscosos $0<\alpha<1$ cos $k T(1+k T)+$

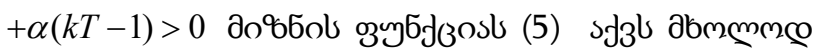

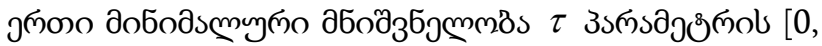

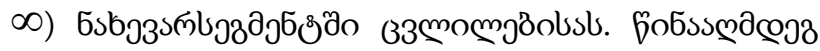

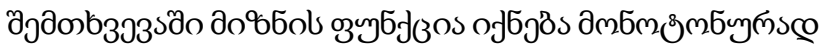

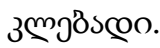

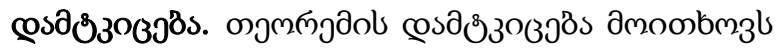

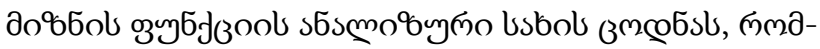

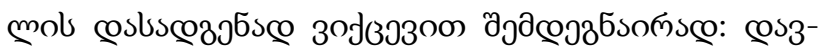

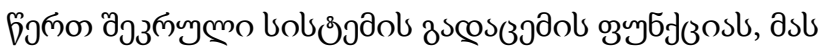
sjals lssbj:

$$
W_{c l}(p)=\frac{k(\tau p+1)}{\alpha T \tau p^{3}+(T+\alpha \tau) p^{2}+(1+k \tau) p+k}
$$

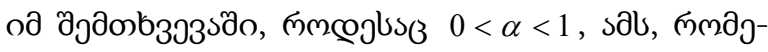

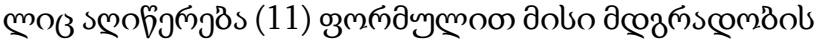

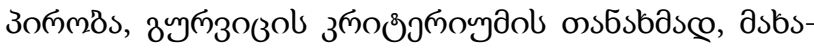

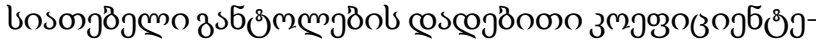

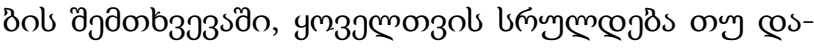

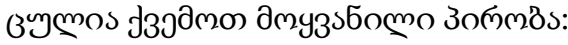

$$
(T+\alpha \tau)(1+k \tau)>\alpha k \tau T
$$

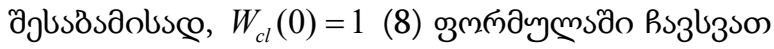

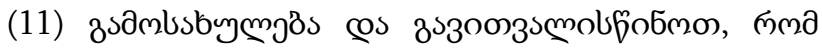
$W_{c l}(0)=1$ дозощјठо):

$$
Y_{m, n}(p)=\frac{\alpha T \tau p^{2}+(T+\alpha \tau) p+1}{\alpha T \tau p^{3}+(T+\alpha \tau) p^{2}+(1+k \tau) p+k} .
$$

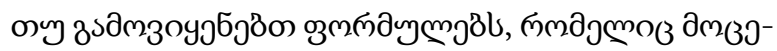

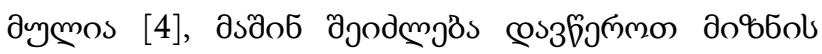

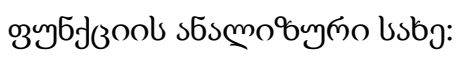

$$
I_{0}=\frac{\left(\alpha T k^{2}+k \alpha^{2}\right) \tau^{2}+(\alpha T k+\alpha)+k T^{2}+T}{2 k^{2} \alpha \tau^{2}+2\left(k \alpha+k^{2} T-K^{2} \alpha T\right) \tau+2 k T}
$$

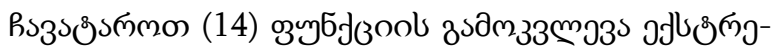

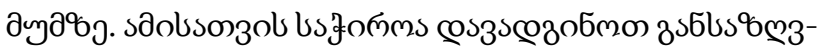

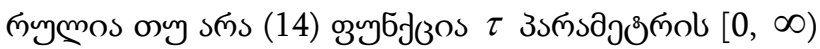

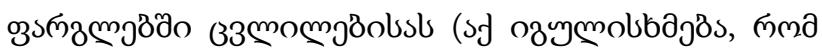

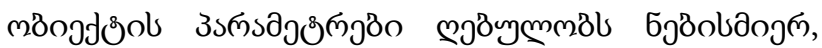




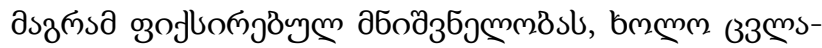

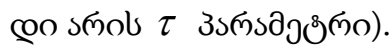

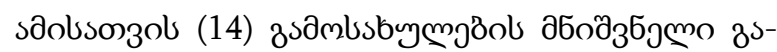

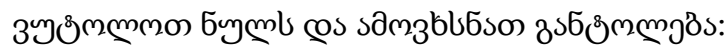

$$
k^{2} \alpha \tau^{2}+k(\alpha+k T-k T \alpha) \tau+k T=0 .
$$

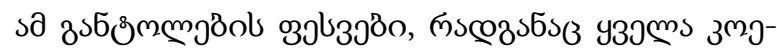

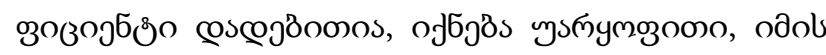

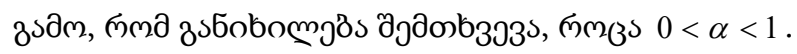

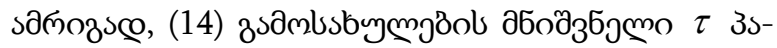

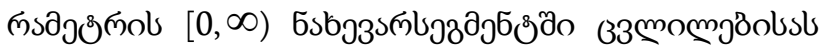

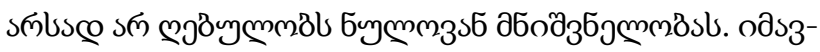

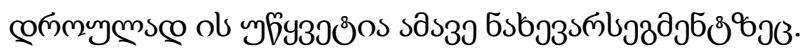

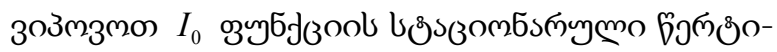

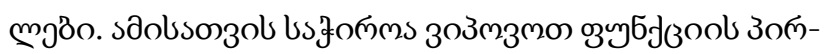

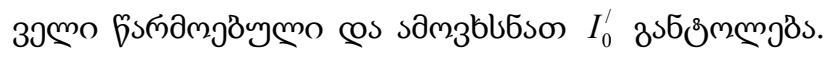
ว3วЈбวฎั, ๓ма

$$
I_{0}^{\prime}=\frac{2 k^{2}(1-\alpha)[k T(1+k T)+\alpha(k T-1)] \tau^{2}-4 k^{2} \alpha T(1-\alpha) \tau-2 k^{2} T^{2}(1+k T)(1-\alpha)}{\left[2 k^{2} \alpha \tau^{2}+2 k(\alpha+k T-k T \alpha) \tau+2 k T\right]^{2}}
$$

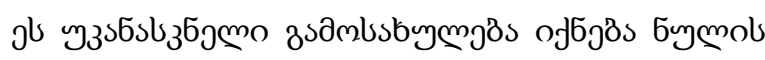

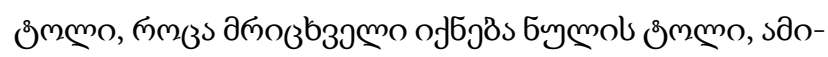

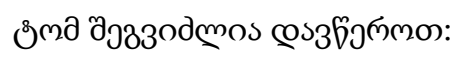

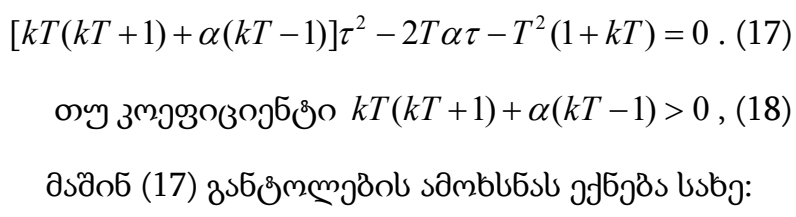

$$
\tau_{1,2}=\frac{T\left(\alpha \pm \sqrt{\alpha^{2}+(k T+1)[k T(k T+1)+\alpha(k T-1)]}\right.}{k T(k T+1)+\alpha(k T-1)}
$$

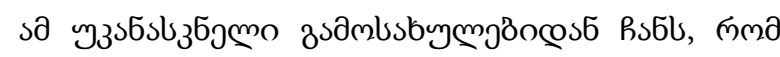

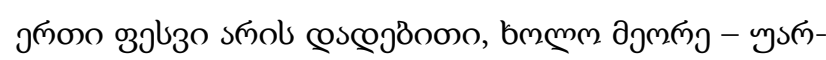
yмовооо.

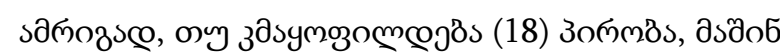

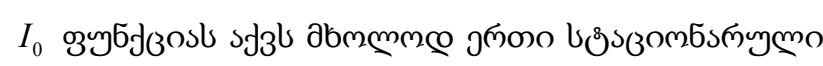

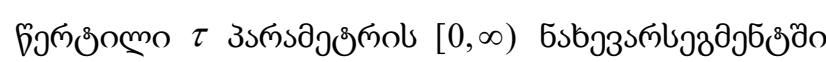

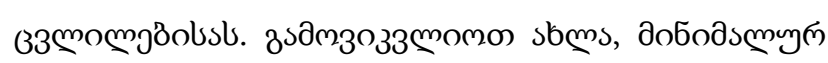

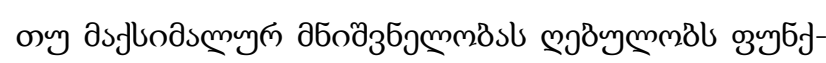

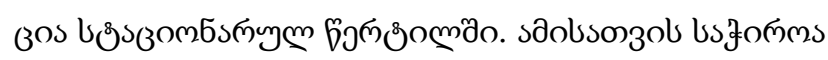

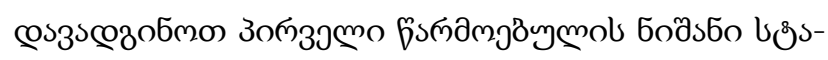

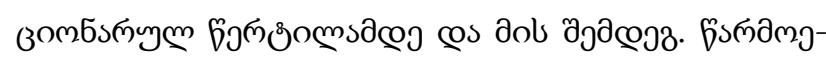

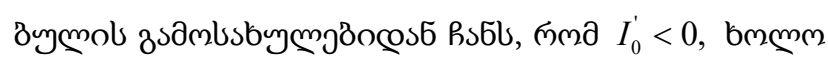

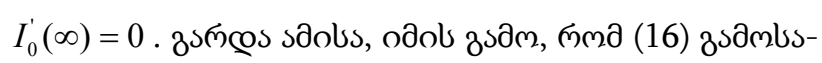

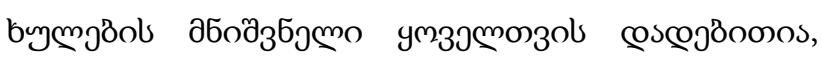

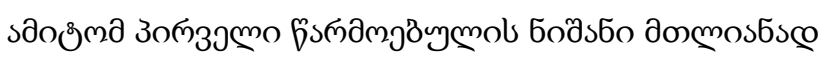

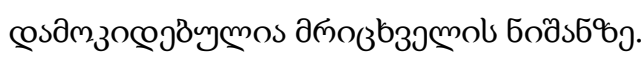

$$
\text { onsolonszsc }
$$

$$
[k T(k T+1)+\alpha(k T-1)] \tau^{2}-2 T \alpha \tau-T^{2}(1+k T)
$$

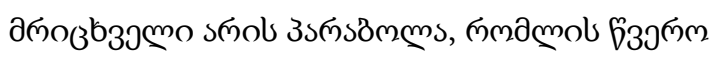

$$
\begin{gathered}
O_{2}\left\{\frac{\alpha T}{k T(1+k T)+\alpha(k T-1)},\right. \\
\left.-2 k^{2} T^{2}(1-\alpha)\left[k T+\frac{\alpha^{2}}{k T(1+k T)+\alpha(k T-1)}\right]\right\}
\end{gathered}
$$

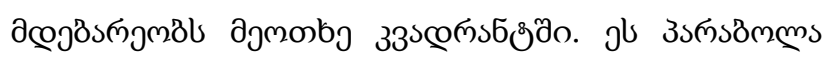

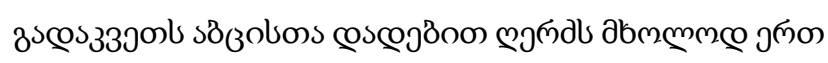

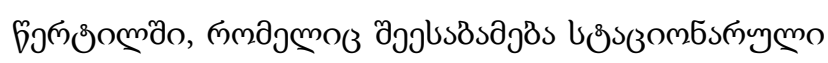

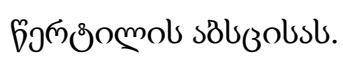

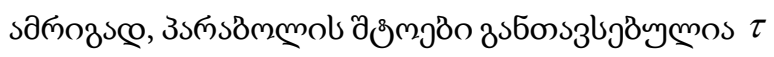

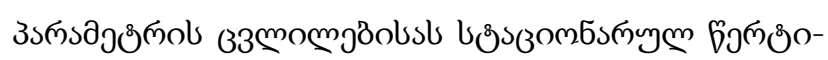

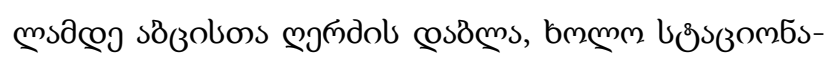

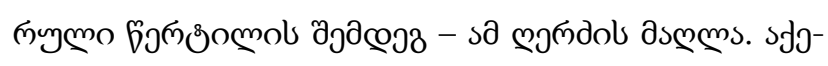

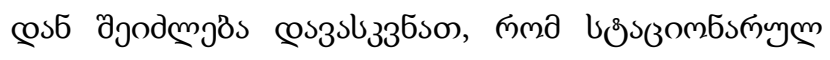




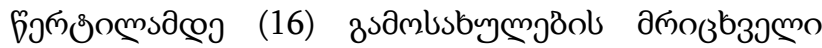

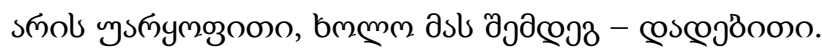

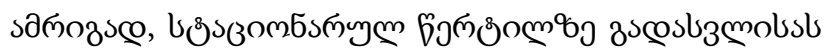

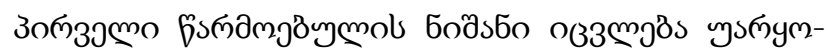

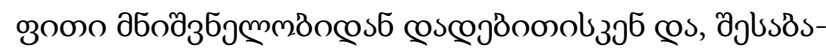

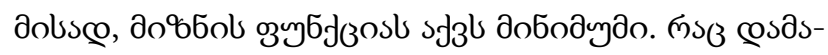

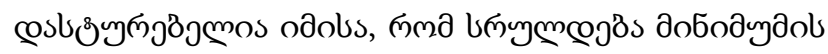

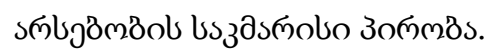

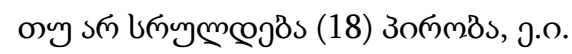

$$
k T(k T+1)+\alpha(k T-1) \leq 0,
$$

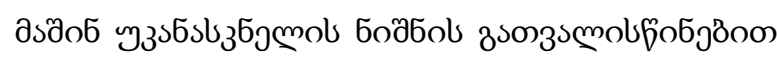

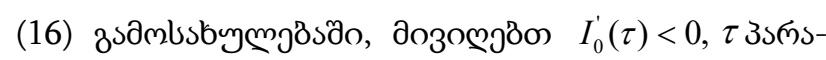

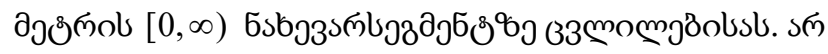

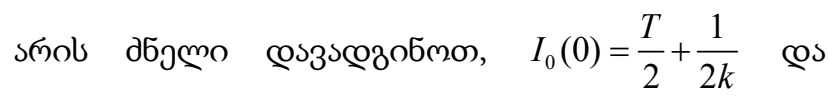

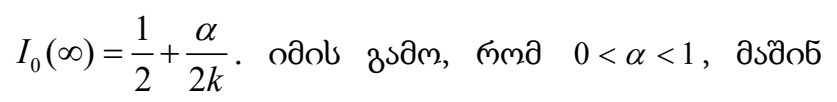

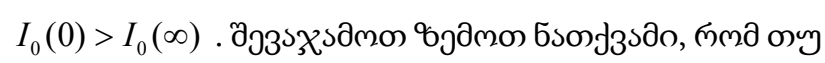

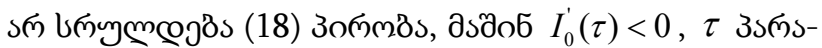

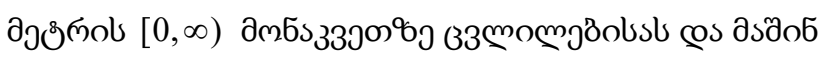

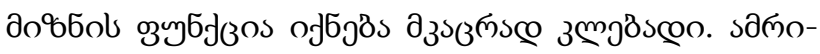

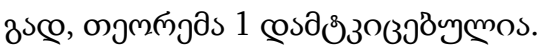

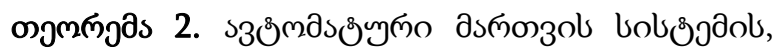

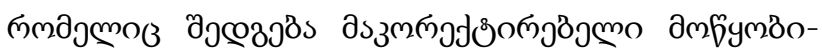

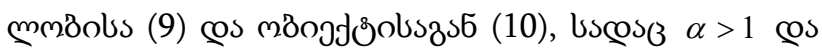

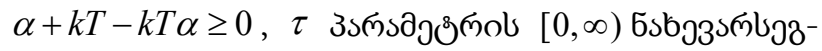

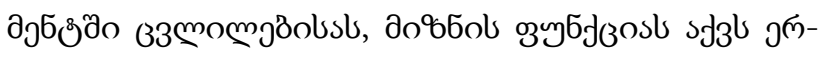

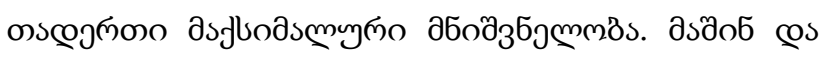

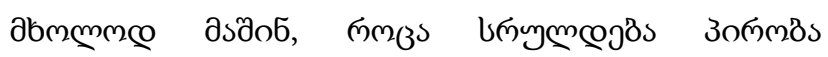
$k T(1+k T)+\alpha(k T-1)>0$.

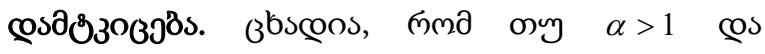

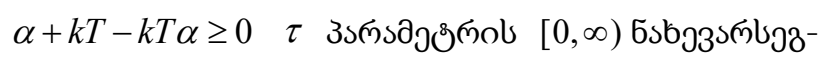

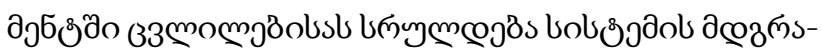

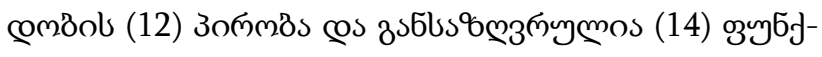
(3os. $I_{0}^{\prime}$-obson 3 ol 33 s $\mathrm{J} 3 \mathrm{b:}$

$$
I_{0}^{\prime}=\frac{-2 k^{2}(\alpha-1)[k T(1+k T)+\alpha(k T-1)] \tau^{2}+4 k^{2} \alpha T(\alpha-1) \tau+2 k^{2} T^{2}(1+k T)(\alpha-1)}{\left[2 k^{2} \alpha \tau^{2}+2 k(\alpha+k T-k T-k T \alpha]^{2}\right.} .
$$

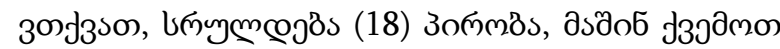

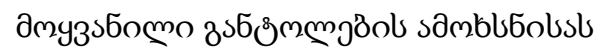

$$
\begin{gathered}
-\left[k T(1+k T 0+\alpha(k T-1)] \tau^{2}+\right. \\
+2 \alpha \tau T+T^{2}(1+k T)=0,
\end{gathered}
$$

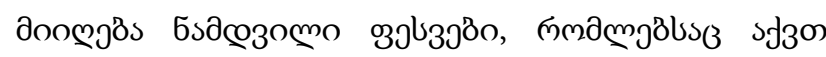

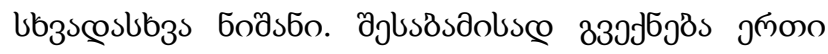

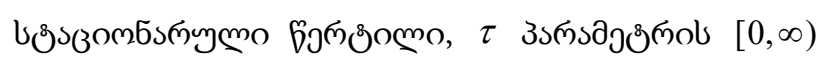

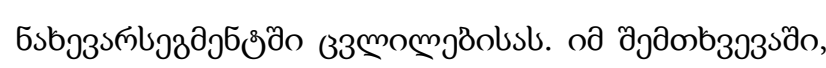

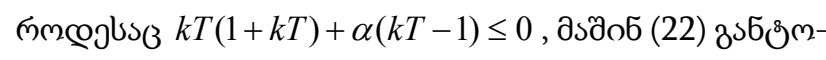

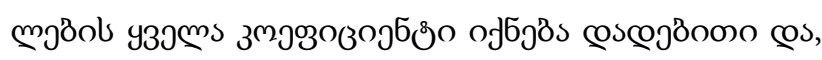

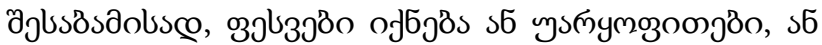

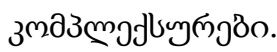

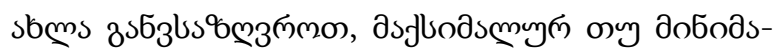

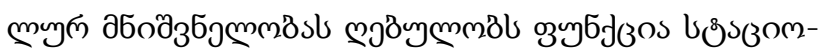

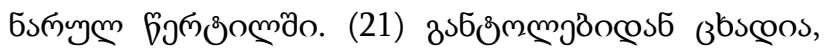

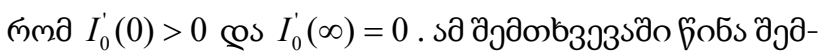

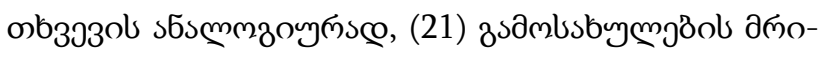

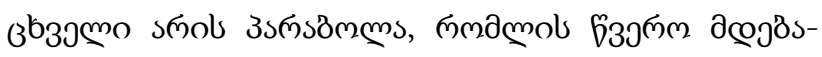

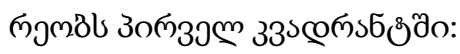




$$
O_{2}\left\{\frac{\alpha T}{k T(1+k T)+\alpha(k T-1)}, 2 k^{2} T^{2}(\alpha-1)\left[1+k T+\frac{\alpha^{2}}{k T(1+k T)+\alpha(k T-1)}\right]\right\}
$$

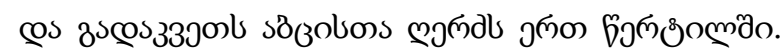

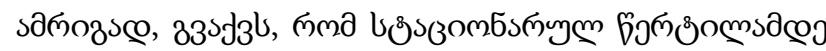

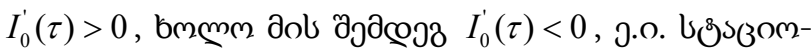

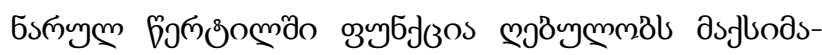

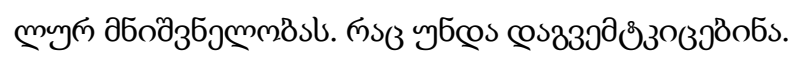

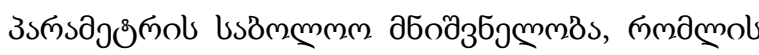

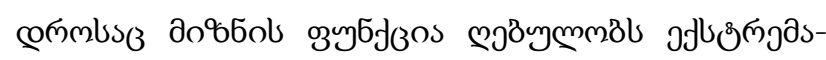

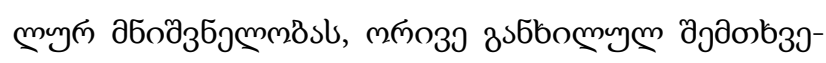

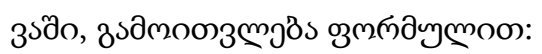

$$
\tau_{1,2}=\frac{T\left\{\alpha+\sqrt{\alpha^{2}+(k T+1)[K T(k T+1)+\alpha(k T-1)]}\right\}}{k T(k T+1)+\alpha(k T-1)} .
$$

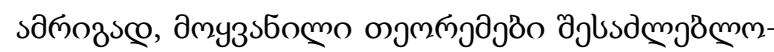

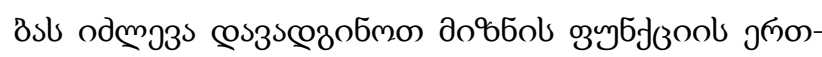

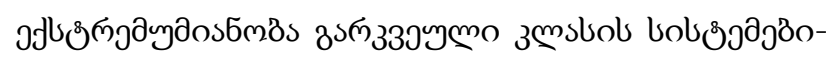

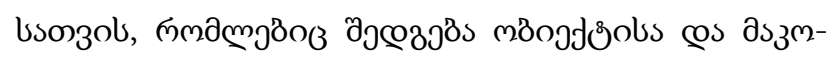

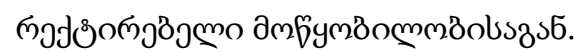

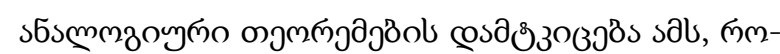

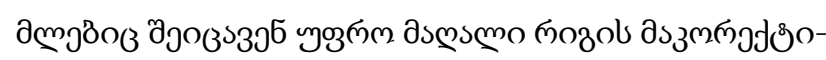

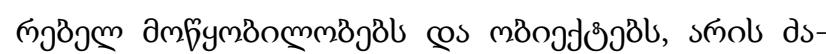

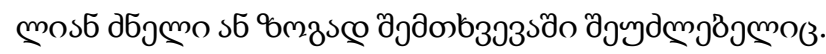

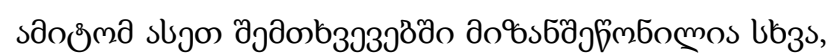

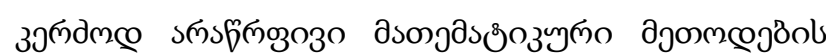

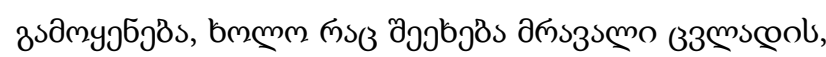

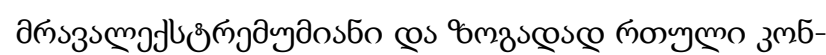

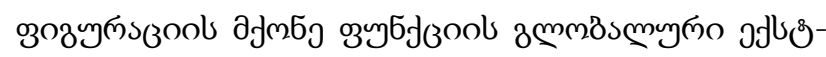

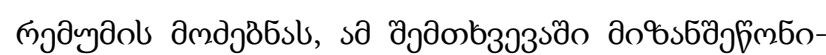

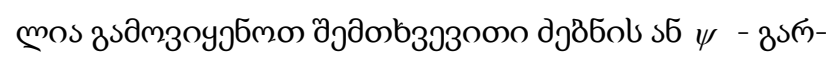

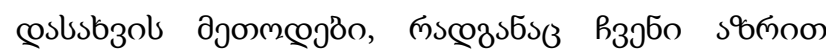

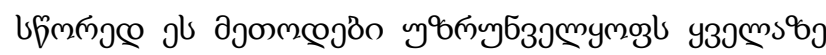

ISSN 1512-0996

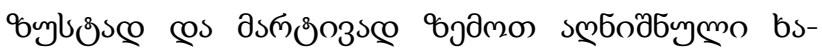

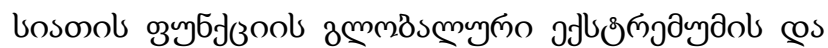

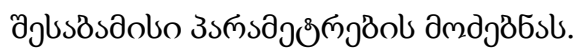

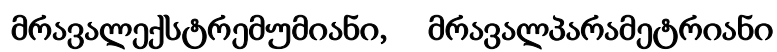

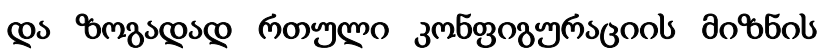

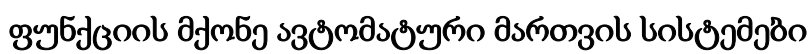

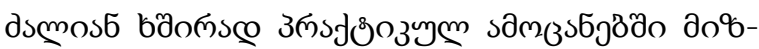
бol озуб

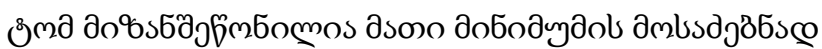

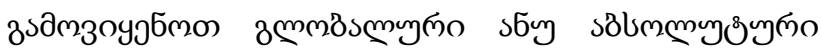

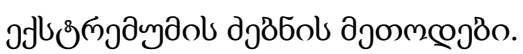

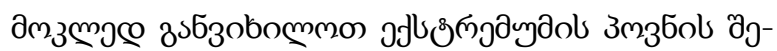

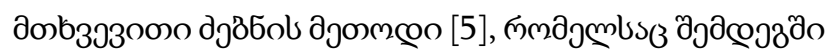

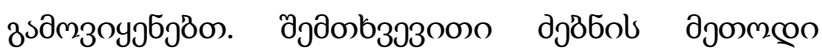

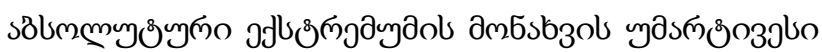

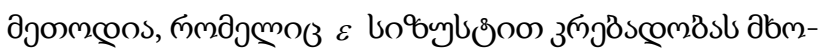

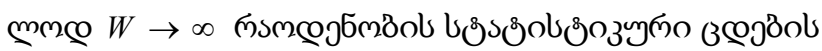

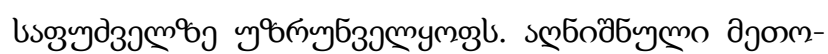

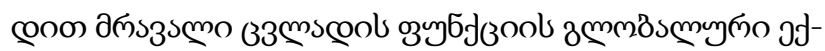

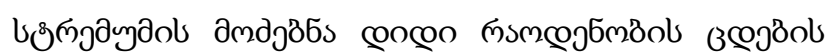

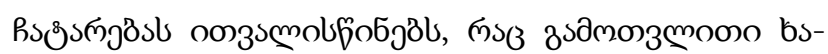

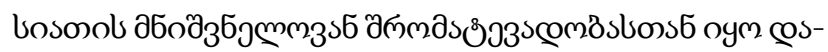

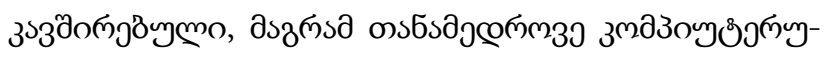

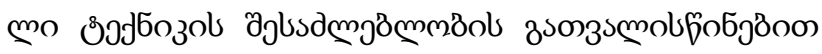

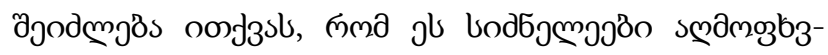
๓о⿴囗十介.

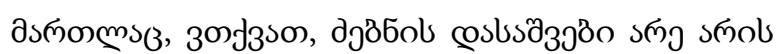

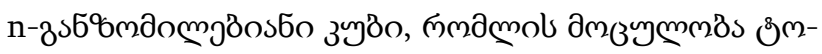

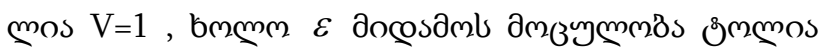

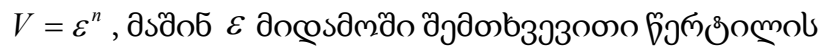




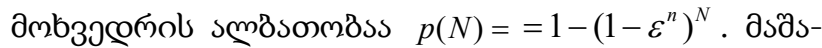

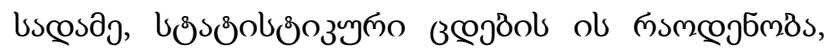

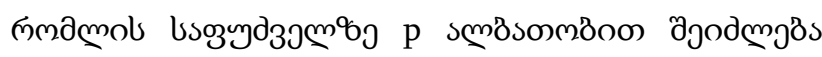
З

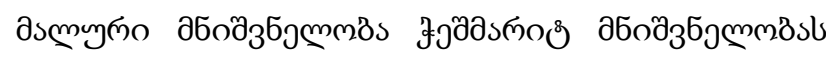

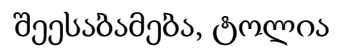

$$
N=\left(\frac{1}{\varepsilon}\right) \cdot \ln [1 /(1-p)] .
$$

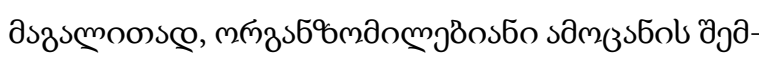

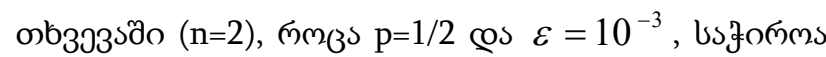

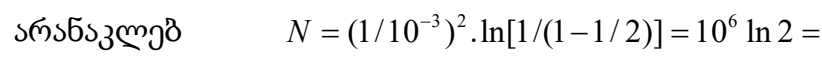

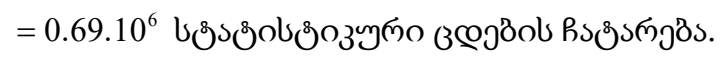

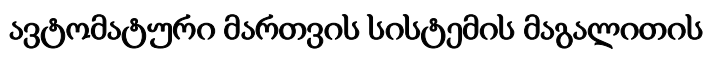

\section{3s6bom3s}

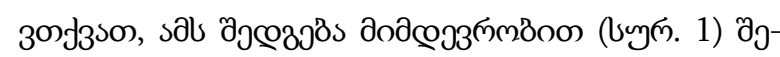

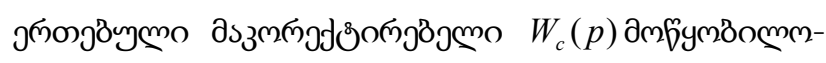

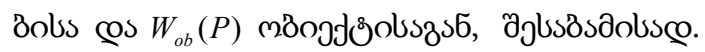

$$
\begin{gathered}
W_{c}(p)=\frac{\left(T_{2} p+1\right)\left(T_{3} p+1\right)}{\left(T_{1} p+1\right)\left(T_{4} p+1\right)}, \\
W_{o b}(p)=\frac{k}{p\left(T_{01} p+1\right)\left(T_{02} p+1\right)\left(T_{03} p+1\right)},
\end{gathered}
$$

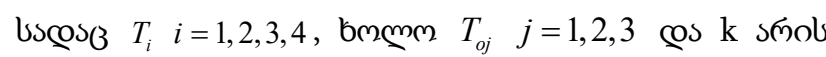

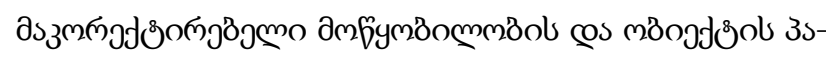

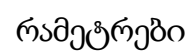

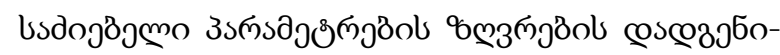

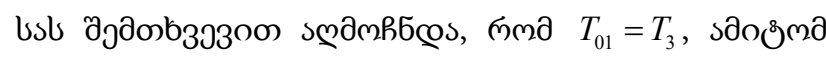

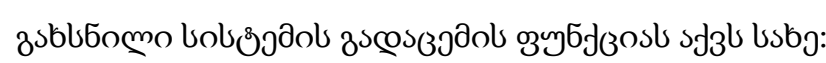

$$
\begin{gathered}
W_{0}(p)=W_{c}(p) \cdot W_{o b}(p)= \\
=\frac{k\left(T_{2} p+1\right)}{p\left(T_{1} p+1\right)\left(T_{4} p+1\right)\left(T_{02} p+1\right)\left(T_{03} p+1\right)},
\end{gathered}
$$

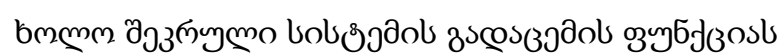
słals bsbo:

$$
\begin{aligned}
& W_{c l}(P)=\frac{W_{o}(p)}{1+W_{o}(p)}= \\
& \frac{k\left(T_{2} p+1\right)}{p\left(T_{1} p+1\right)\left(T_{4} p+1\right)\left(T_{02} p+1\right)\left(T_{03} p+1\right)+k\left(T_{2} p+1\right)} .
\end{aligned}
$$

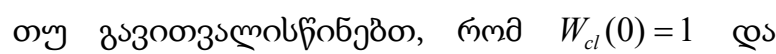

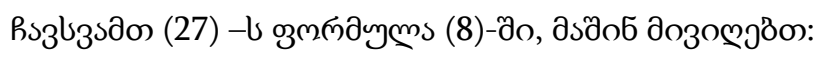

$$
\begin{gathered}
Y_{m, n}(p)=\frac{1}{p}[1- \\
\left.\frac{k\left(T_{2} p+1\right)}{p\left(T_{1} p+1\right)\left(T_{4} p+1\right)\left(T_{02} p+1\right)\left(T_{03} p+1\right)+k\left(T_{2} p+1\right)}\right]= \\
=\frac{\left[T_{1} T_{4} p^{2}+\left(T_{1}+T_{4}\right) p+1\right]\left[T_{02} T_{03} p^{2}+\left(T_{02}+T_{03}\right) p+1\right]}{p\left\{T_{1} T_{4} p^{2}+\left(T_{1}+T_{4}\right) p+1\right]\left[T_{02} T_{03} p^{2}+\left(T_{02}+T_{03}\right) p+1\right\}+k T_{2} p+k} .
\end{gathered}
$$

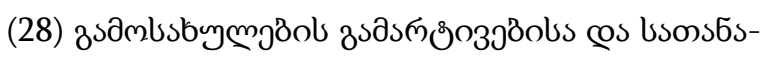

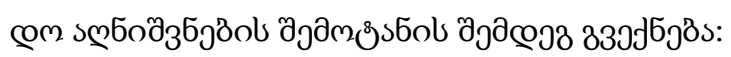

$$
Y_{m, n}(p)=\frac{b_{4} p^{4}+b_{3} p^{3}+b_{2} p^{2}+b_{1} p+b_{0}}{c_{5} p^{5}+c_{4} p^{4}+c_{3} p^{3}+c_{2} p^{2}+c_{1} p+c_{0}}
$$

uscosos

$$
\begin{aligned}
& c_{5}=b_{4}=T_{1} T_{4} T_{02} T_{03} \\
& c_{4}=b_{3}=T_{1} T_{4} T_{02}+T_{1} T_{4} T_{03}+T_{1} T_{02} T_{03}+T_{4} T_{02} T_{03} \\
& c_{3}=b_{2}=T_{1} T_{4}+T_{1} T_{02}+T_{1} T_{03}+T_{4} T_{02}+T_{4} T_{03}+T_{02} T_{03} \\
& c_{2}=b_{1}=T_{1}+T_{4}+T_{02}+T_{03} \\
& c_{1}=1+k T_{2} \\
& c_{0}=k \\
& b_{0}=1
\end{aligned}
$$

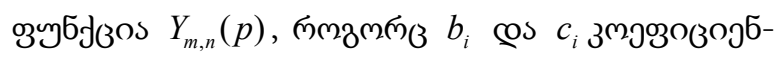

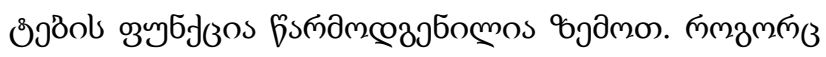

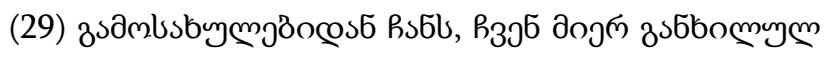

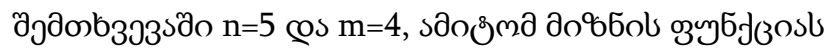

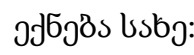

$$
I_{0}=\frac{1}{\Delta}\left[b_{4}^{2} m_{0}+\left(b_{3}^{2}+2 b_{2} b_{4}\right) m_{1}+\left(b_{2}^{2}-2 b_{1} b_{3}+2 b_{0} b_{4}\right) m_{2}+\right.
$$

$$
\left.+\left(b_{1}^{2}-2 b_{0} b_{2}\right) m_{3}+b_{0}^{2} m_{4}\right]
$$


uscosos

$$
\begin{aligned}
& m_{1}=-c_{0} c_{3}+c_{1} c_{2}, \\
& m_{0}=\frac{1}{c_{5}}\left(c_{3} m_{1}-c_{1} m_{2}\right), \\
& m_{2}=-c_{0} c_{5}+c_{1} c_{4}, \\
& m_{3}=\frac{1}{c_{0}}\left(c_{2} m_{2}-c_{4} m_{1}\right), \\
& m_{4}=\frac{1}{c_{0}}\left(c_{2} m_{3}-c_{4} m_{2}\right), \\
& \Delta=c_{0}\left(c_{1} m_{4}-c_{3} m_{3}+c_{5} m_{4}\right)
\end{aligned}
$$

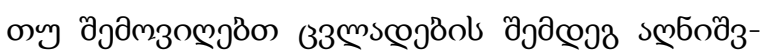

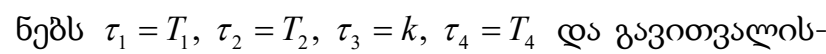

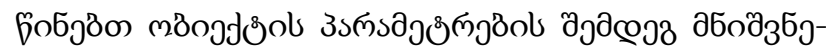

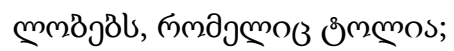

$$
\begin{aligned}
& T_{02}=0.02 \mathrm{sec} \\
& T_{03}=0.01 \mathrm{sec}
\end{aligned}
$$

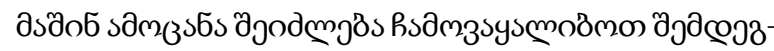
Бsońsc:

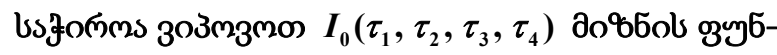

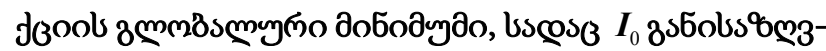

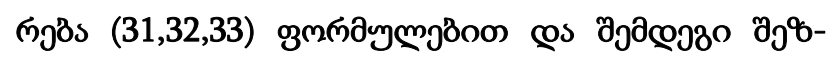

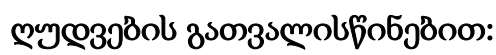

$$
\begin{aligned}
& 2.27 \leq \tau_{1} \leq 10, \\
& 0.125 \leq \tau_{2} \leq 0.5, \\
& 200 \leq \tau_{3} \leq 225, \\
& 0.02 \leq \tau_{4} \leq 0.03,
\end{aligned}
$$

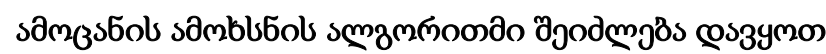

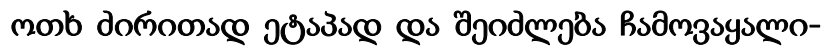

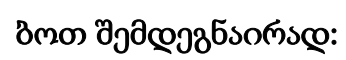

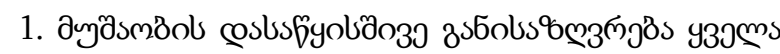

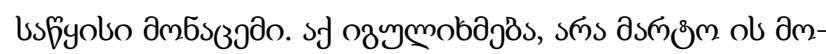

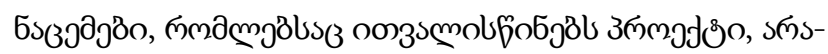

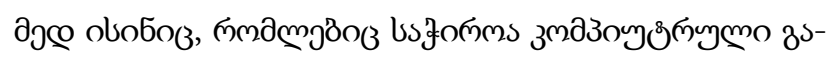

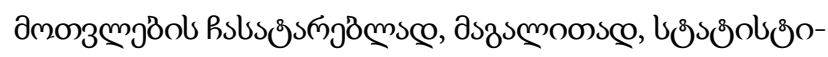

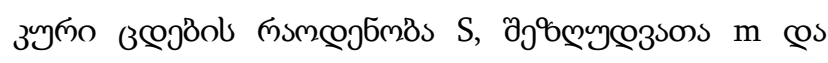

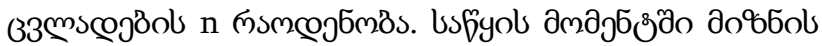

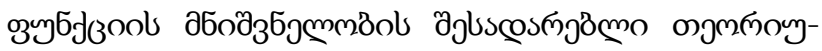
msce ajlusdmm coneo nogbzol boconco $-I_{\text {min }}$.

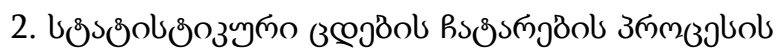

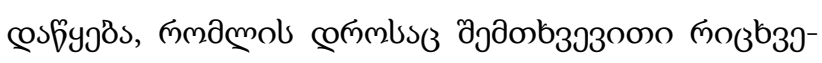

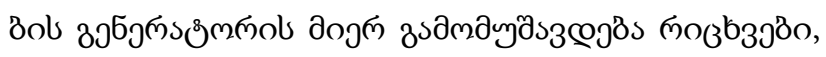

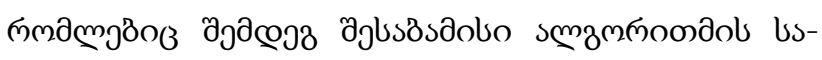

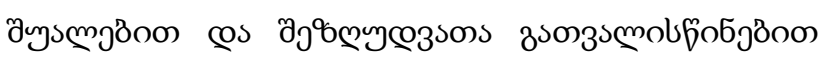

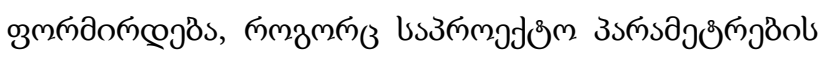

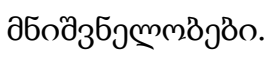

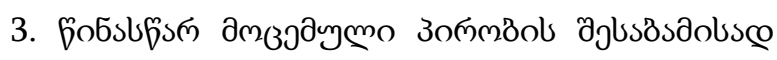

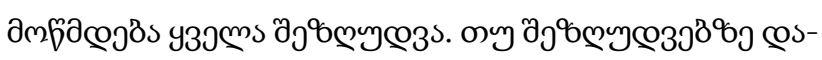

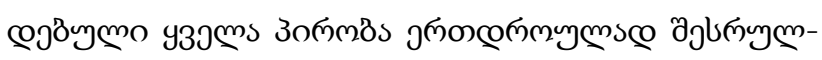

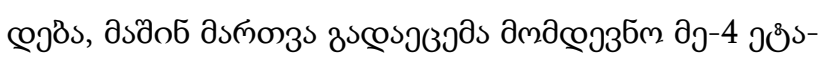

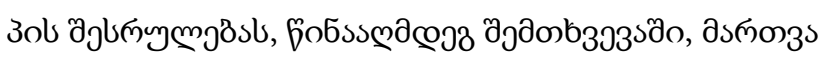

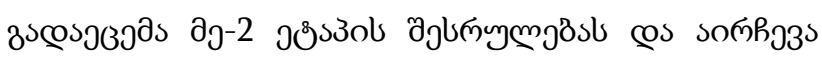

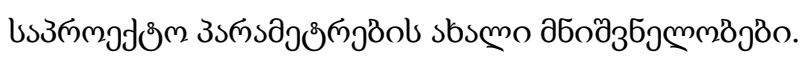

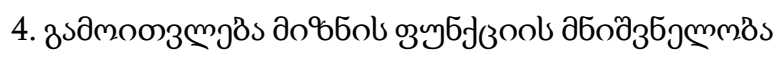

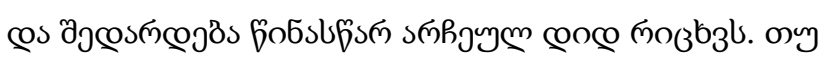

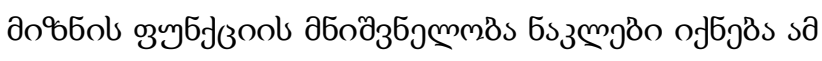

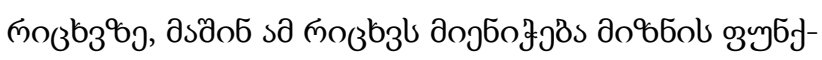

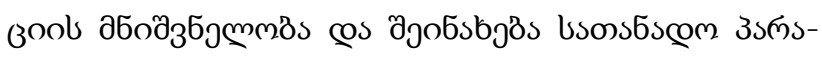

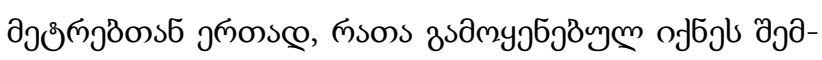

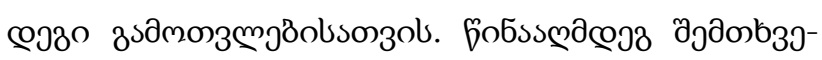

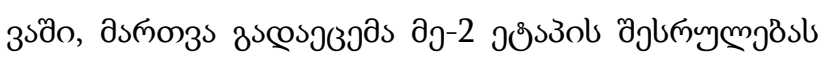

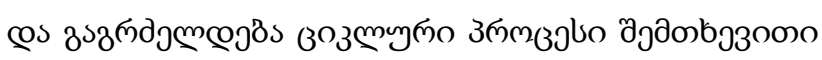

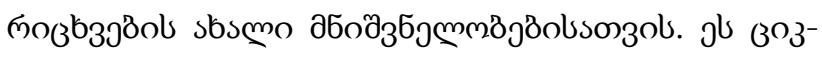

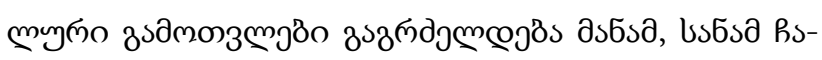

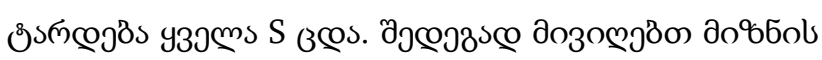

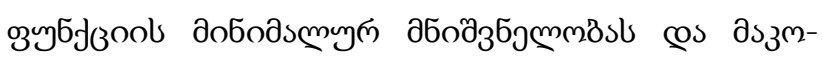

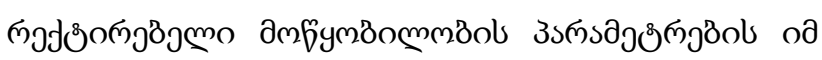

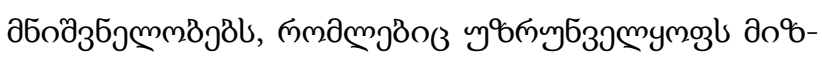

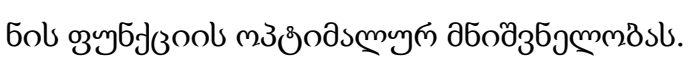




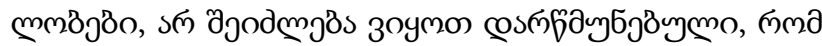

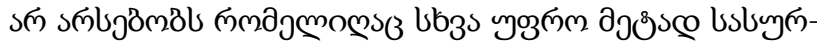

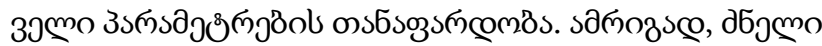

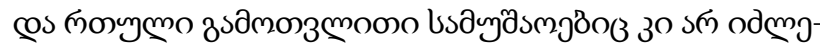

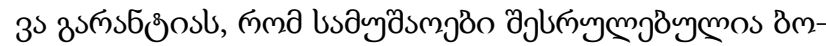

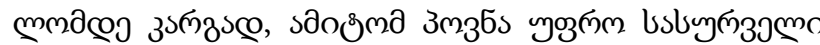

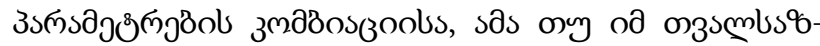

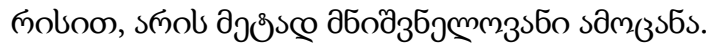

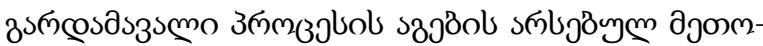

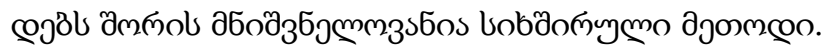

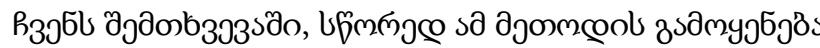

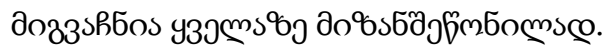

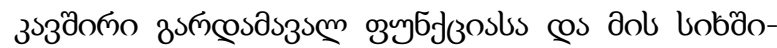

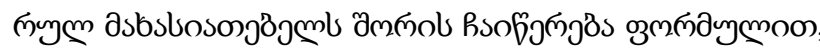

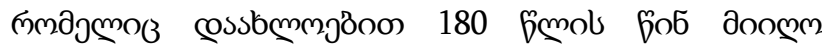

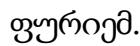

$$
y(t)=\frac{2}{\pi} \int_{0}^{\infty} \frac{U(\omega)}{\omega} \sin (\omega t) d \omega
$$

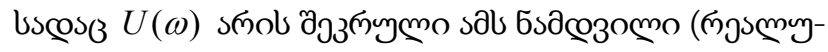

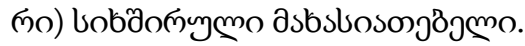

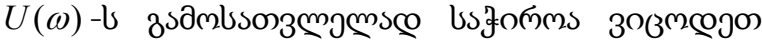

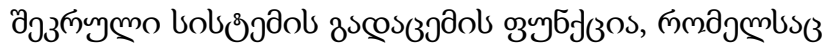
sj3bl bsbo:

$$
\begin{gathered}
W_{c l}(p)=\frac{W_{o p}(p)}{1+W_{o p}(p)}= \\
=\frac{k\left(T_{2} p+1\right)}{P(T p+1)\left(T_{4} p+1\right)\left(T_{02} p+1\right)\left(T_{03} p+1\right)+k\left(T_{2} p+1\right)}
\end{gathered}
$$

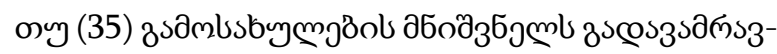

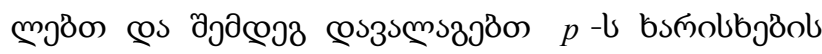

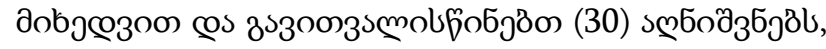

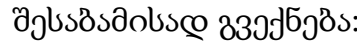

$$
W_{c l}(p)=\frac{k\left(T_{2} p+1\right)}{c_{5} p^{5}+c_{4} p^{4}+c_{3} p^{3}+c_{2} p^{2}+c_{1} p+c}
$$

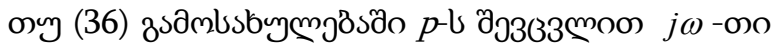

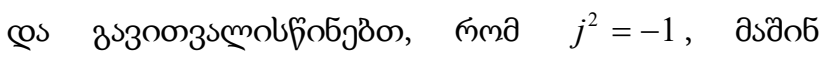
33อ」6วठs:

$$
\begin{gathered}
W_{c l}(j \omega)= \\
=\frac{k\left(T_{2} j \omega+1\right)}{j c_{5} \omega^{5}+c_{4} \omega^{4}-j c_{3} \omega^{3}-c_{2} \omega^{2}+j c_{1} \omega+c_{0}}= \\
=\frac{k\left(T_{2} j \omega+1\right)}{\left(c_{4} \omega^{4}-c_{2} \omega^{2}+c_{0}\right)+j\left(c_{5} \omega^{5}-c_{3} \omega^{3}+c_{1} \omega\right)}
\end{gathered}
$$

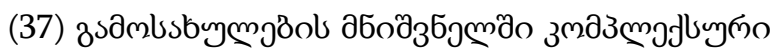

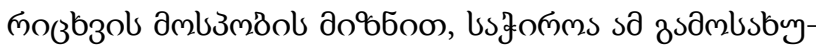

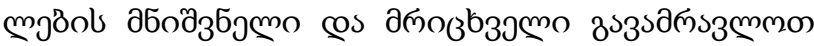

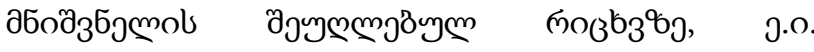

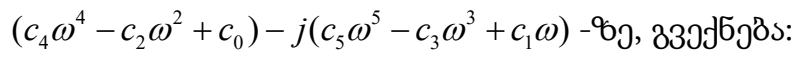

$$
W_{c l}(j \omega)=
$$

$=\frac{k\left(j T_{2} \omega+1\right)\left[\left(c_{4} \omega^{4}-c_{2} \omega^{2}+c_{0}\right)-j\left(c_{5} \omega^{5}-c_{3} \omega^{3}+c_{1} \omega\right]\right.}{\left(c_{4} \omega^{4} \omega-c_{2} \omega^{2}+c_{0}\right)^{2}+\left(c_{5} \omega^{5}-c_{3} \omega^{3}+c_{1} \omega\right)^{2}}$

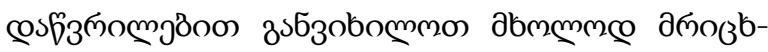

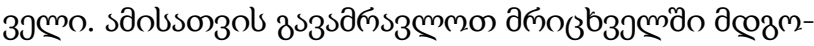

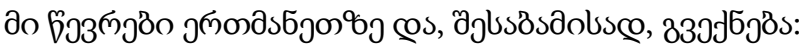

$$
\begin{gathered}
k\left[j T_{2} \omega\left(c_{4} \omega^{4}-c_{2} \omega^{2}+c_{0}\right)+T_{2} \omega\left(c_{5} \omega^{5}-c_{3} \omega^{3}+c_{1} \omega\right)+\right. \\
\left.+\left(c_{4} \omega^{4}-c_{2} \omega^{2}+c_{0}\right)-j\left(c_{5} \omega^{5}-c_{3} \omega^{3}+c_{1} \omega\right)\right]= \\
=k\left[T_{2} \omega\left(c_{5} \omega^{5}-c_{3} \omega^{3}+c_{1} \omega\right)+\left(c_{4} \omega^{4}-c_{2} \omega^{2}+c_{0}\right)\right]+ \\
\left.\quad+j k\left[c_{4} \omega^{4}-c_{2} \omega^{2}+c_{0}\right)-\left(c_{5} \omega^{5}-c_{3} \omega^{3}+c_{1} \omega\right)\right]
\end{gathered}
$$

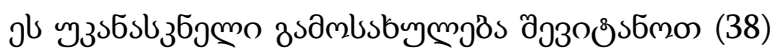

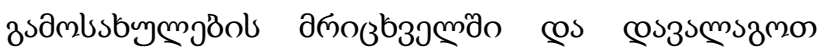

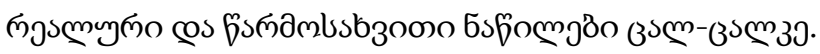
วзЈбวฎัs:

$$
W_{c l}(j \omega)=U(\omega)+j V(\omega),
$$

uscosos

$$
\begin{gathered}
U(\omega)=\frac{k\left[T_{2} \omega\left(c_{5} \omega^{5}-c_{3} \omega^{3}+c_{1} \omega\right)+\left(c_{4} \omega^{4}-c_{2} \omega^{2}+c_{0}\right)\right]}{\left(c_{4} \omega^{4}-c_{2} \omega^{2}+c_{0}\right)^{2}+\left(c_{5} \omega^{5}-c_{3} \omega^{3}+c_{1} \omega\right)^{2}}, \\
V(\omega)=j \frac{k\left[T_{2} \omega\left(c_{4} \omega^{4}-c_{2} \omega^{2}+c_{0}\right)-\left(c_{5} \omega^{5}-c_{3} \omega^{3}+c_{1} \omega\right)\right]}{\left(c_{4} \omega^{4}-c_{2} \omega^{2}+c_{0}\right)^{2}+\left(c_{5} \omega^{5}-c_{3} \omega^{3}+c_{1} \omega\right)}
\end{gathered}
$$




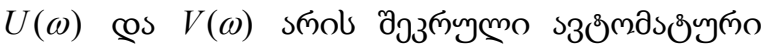

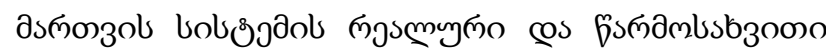

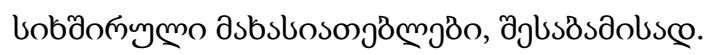

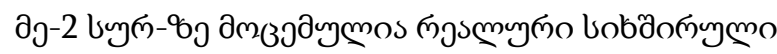
asbsbosonğmol zю̆sозо

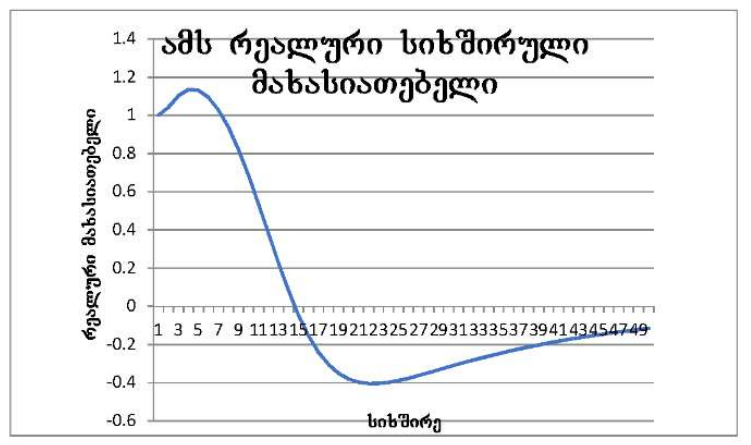

๒งyn. 2.

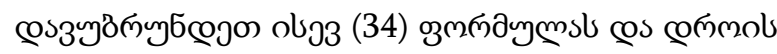

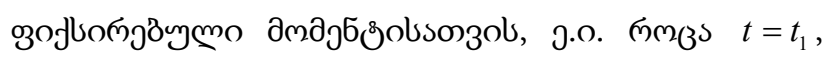

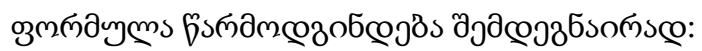

$$
y\left(t_{1}\right)=\frac{2}{\pi} \int_{0}^{\omega_{l}} U(\omega) \frac{\operatorname{Sin}\left(\omega t_{1}\right)}{\omega} d \omega .
$$

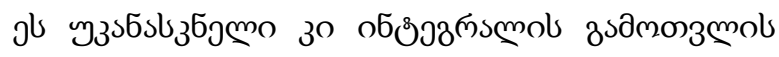

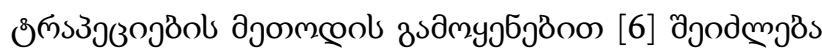

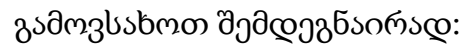

$$
\begin{gathered}
y\left(t_{1}\right)=\frac{2}{\pi} \Delta \omega\left[\frac{1}{2} \frac{U\left(\omega_{1}\right)}{\omega_{1}} \operatorname{Sin}\left(t_{1} \omega_{1}\right)+\frac{U\left(\omega_{2}\right)}{\omega_{2}} \operatorname{Sin}\left(t_{1} \omega_{2}\right)+, \ldots,+\right. \\
\left.+\frac{U\left(\omega_{l-l}\right)}{\omega_{l-1}} \operatorname{Sin}\left(t_{1} \omega_{l-1}\right)+\frac{1}{2} \frac{U\left(\omega_{l}\right)}{\omega_{l}} \operatorname{Sin}\left(t_{1} \omega_{l}\right)\right]= \\
=\frac{2}{\pi} \Delta \omega\left\{\sum_{i=2}^{l-1} \frac{U\left(\omega_{i}\right)}{\omega_{i}} \operatorname{Sin}\left(t_{1} \omega_{i}\right)+\right. \\
\left.+0.5\left[\frac{U\left(\omega_{1}\right)}{\omega_{1}} \operatorname{Sin}\left(t_{1} \omega_{1}\right)+\frac{U\left(\omega_{l}\right)}{\omega_{l}} \operatorname{Sin}\left(t_{1} \omega_{l}\right)\right]\right\}
\end{gathered}
$$

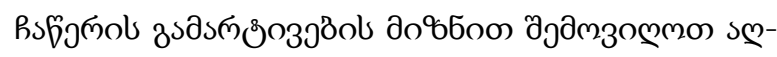
ธoð3ธs:

$$
\frac{U(\omega)}{\omega} \operatorname{Sin}(\omega t)=z
$$

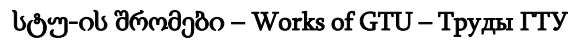

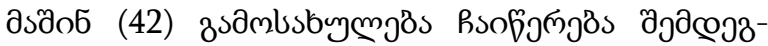
5somsc:

$$
y\left(t_{i}\right)=\frac{2}{\pi} \Delta \omega\left[\sum_{i=2}^{\ell-1} z_{i j}+0.5\left(z_{1 j}+z_{\ell j}\right)\right], j=1,2, \ldots, M,
$$

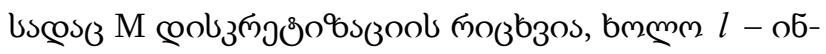

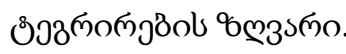

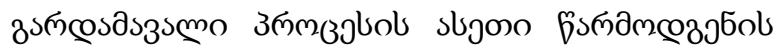

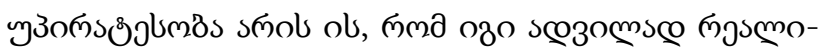

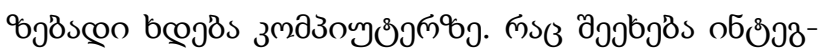

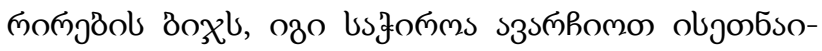

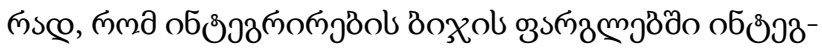

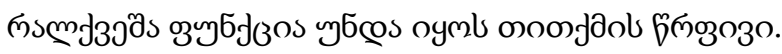

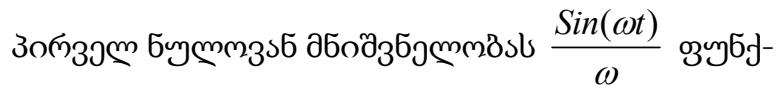

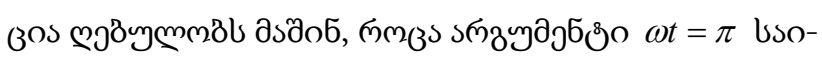

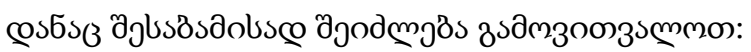

$$
\omega=\frac{\pi}{t_{r}}
$$

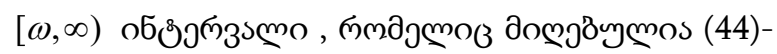

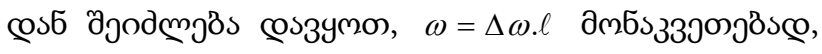

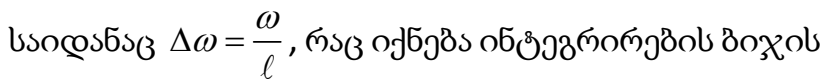

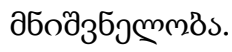

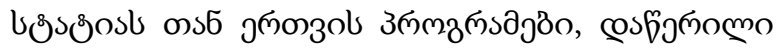

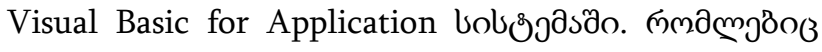

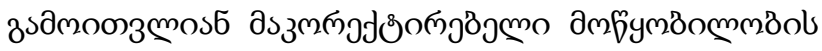

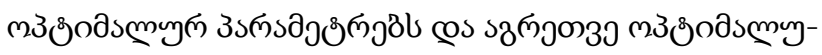

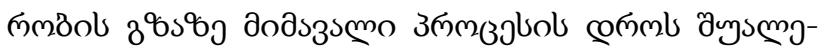

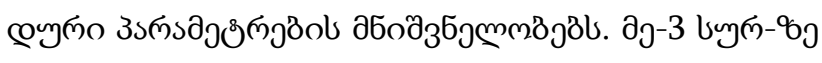

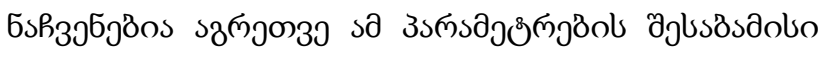

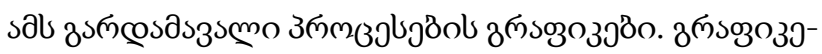

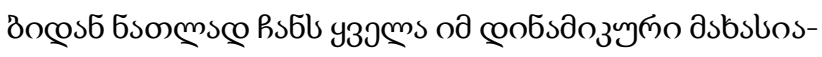

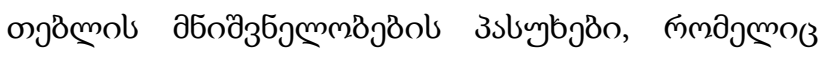
cosol3s sab lobogkob coslusfyolano. 
उbromo 2

\begin{tabular}{|c|c|c|c|c|}
\hline $\mathrm{t}$ & $\mathrm{y} 1(\mathrm{t})$ & $\mathrm{y} 2(\mathrm{t} 0$ & $y 3(t)$ & $y 4(t)$ \\
\hline 0.01 & $7.80 \mathrm{E}-05$ & 0.000392 & 0.000185 & $8.83 \mathrm{E}-05$ \\
\hline 0.02 & 0.000195 & 0.000932 & 0.000445 & 0.000218 \\
\hline 0.03 & 0.000387 & 0.001739 & 0.000844 & 0.000426 \\
\hline 0.04 & 0.000678 & 0.002889 & 0.001429 & 0.000738 \\
\hline 0.05 & 0.001085 & 0.004396 & 0.00222 & 0.00117 \\
\hline 0.06 & 0.001611 & 0.006211 & 0.003211 & 0.001725 \\
\hline 0.07 & 0.002248 & 0.008232 & 0.004371 & 0.002392 \\
\hline 0.08 & 0.002978 & 0.01031 & 0.005651 & 0.003151 \\
\hline 0.09 & 0.003777 & 0.01228 & 0.006985 & 0.003975 \\
\hline 0.1 & 0.004618 & 0.013982 & 0.008308 & 0.004834 \\
\hline 0.11 & 0.005474 & 0.015281 & 0.009559 & 0.005698 \\
\hline 0.12 & 0.006321 & 0.016091 & 0.010687 & 0.006542 \\
\hline 0.13 & 0.007141 & 0.016379 & 0.011658 & 0.007349 \\
\hline 0.14 & 0.007923 & 0.016168 & 0.012457 & 0.008105 \\
\hline 0.15 & 0.00866 & 0.015534 & 0.013083 & 0.008807 \\
\hline 0.16 & 0.00935 & 0.014587 & 0.01355 & 0.009454 \\
\hline 0.17 & 0.009995 & 0.013457 & 0.013877 & 0.010049 \\
\hline 0.18 & 0.010598 & 0.012275 & 0.01409 & 0.010598 \\
\hline 0.19 & 0.011161 & 0.011158 & 0.014209 & 0.011104 \\
\hline 0.2 & 0.011683 & 0.010194 & 0.014252 & 0.011569 \\
\hline 0.21 & 0.012165 & 0.009442 & 0.014231 & 0.011993 \\
\hline 0.22 & 0.012602 & 0.008928 & 0.014154 & 0.012373 \\
\hline 0.23 & 0.012993 & 0.008647 & 0.014023 & 0.012708 \\
\hline 0.24 & 0.013333 & 0.008577 & 0.013843 & 0.012993 \\
\hline 0.25 & 0.013621 & 0.008682 & 0.013617 & 0.013229 \\
\hline 0.26 & 0.013856 & 0.008923 & 0.013353 & 0.013414 \\
\hline 0.27 & 0.014042 & 0.009262 & 0.013063 & 0.013553 \\
\hline 0.28 & 0.014182 & 0.009668 & 0.01276 & 0.013651 \\
\hline 0.29 & 0.014283 & 0.010114 & 0.012461 & 0.013714 \\
\hline 0.3 & 0.014351 & 0.010578 & 0.012182 & 0.013751 \\
\hline 0.31 & 0.014393 & 0.011039 & 0.011936 & 0.013766 \\
\hline 0.32 & 0.014412 & 0.011476 & 0.011731 & 0.013767 \\
\hline
\end{tabular}




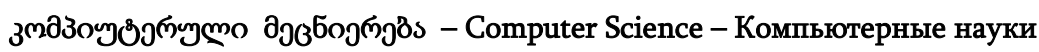

\begin{tabular}{|c|c|c|c|c|}
\hline 0.33 & 0.014413 & 0.011869 & 0.01157 & 0.013757 \\
\hline 0.34 & 0.014398 & 0.012194 & 0.011452 & 0.013736 \\
\hline 0.35 & 0.014366 & 0.01243 & 0.01137 & 0.013706 \\
\hline 0.36 & 0.014319 & 0.012562 & 0.011318 & 0.013665 \\
\hline 0.37 & 0.014255 & 0.012581 & 0.011285 & 0.013613 \\
\hline 0.38 & 0.014175 & 0.012491 & 0.011265 & 0.013549 \\
\hline 0.39 & 0.01408 & 0.012305 & 0.011252 & 0.013474 \\
\hline 0.4 & 0.013973 & 0.012048 & 0.011246 & 0.013391 \\
\hline 0.41 & 0.013858 & 0.011752 & 0.011248 & 0.013302 \\
\hline 0.42 & 0.013738 & 0.011454 & 0.01126 & 0.013211 \\
\hline 0.43 & 0.013618 & 0.011187 & 0.011286 & 0.013123 \\
\hline 0.44 & 0.013501 & 0.010979 & 0.011328 & 0.013041 \\
\hline 0.45 & 0.013388 & 0.010846 & 0.011386 & 0.012966 \\
\hline 0.46 & 0.013282 & 0.010794 & 0.011458 & 0.012899 \\
\hline 0.47 & 0.013182 & 0.010817 & 0.011538 & 0.01284 \\
\hline 0.48 & 0.013086 & 0.0109 & 0.01162 & 0.012786 \\
\hline 0.49 & 0.012994 & 0.011023 & 0.011698 & 0.012736 \\
\hline 0.5 & 0.012904 & 0.011166 & 0.011766 & 0.012687 \\
\hline 0.51 & 0.012816 & 0.01131 & 0.011819 & 0.012638 \\
\hline 0.52 & 0.012729 & 0.011442 & 0.011857 & 0.012589 \\
\hline 0.53 & 0.012644 & 0.011555 & 0.011882 & 0.012542 \\
\hline 0.54 & 0.012563 & 0.01165 & 0.011896 & 0.012496 \\
\hline 0.55 & 0.012489 & 0.01173 & 0.011906 & 0.012455 \\
\hline 0.56 & 0.012422 & 0.0118 & 0.011916 & 0.01242 \\
\hline 0.57 & 0.012363 & 0.011866 & 0.011929 & 0.012393 \\
\hline 0.58 & 0.012315 & 0.011928 & 0.011949 & 0.012373 \\
\hline 0.59 & 0.012275 & 0.011986 & 0.011973 & 0.012361 \\
\hline 0.6 & 0.012243 & 0.012035 & 0.012 & 0.012355 \\
\hline 0.61 & 0.012216 & 0.012067 & 0.012026 & 0.012353 \\
\hline 0.62 & 0.012194 & 0.012078 & 0.012048 & 0.012352 \\
\hline 0.63 & 0.012174 & 0.012064 & 0.012063 & 0.012351 \\
\hline 0.64 & 0.012155 & 0.012026 & 0.012069 & 0.012349 \\
\hline 0.65 & 0.012138 & 0.011968 & 0.012067 & 0.012345 \\
\hline 0.66 & 0.012122 & 0.011901 & 0.012059 & 0.012341 \\
\hline
\end{tabular}




\begin{tabular}{|c|c|c|c|c|}
\hline 0.67 & 0.012109 & 0.011836 & 0.01205 & 0.012338 \\
\hline 0.68 & 0.0121 & 0.011787 & 0.012044 & 0.012337 \\
\hline 0.69 & 0.012096 & 0.011762 & 0.012043 & 0.01234 \\
\hline 0.7 & 0.012098 & 0.011768 & 0.012051 & 0.012347 \\
\hline 0.71 & 0.012105 & 0.011804 & 0.012068 & 0.012359 \\
\hline 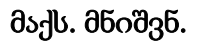 & $1.44 \mathrm{E}-02$ & 0.016379 & 0.014252 & $1.38 \mathrm{E}-02$ \\
\hline
\end{tabular}

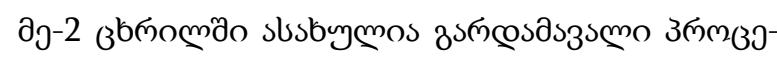

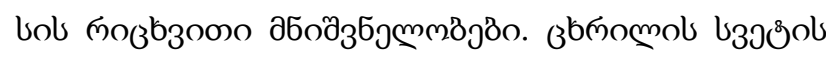

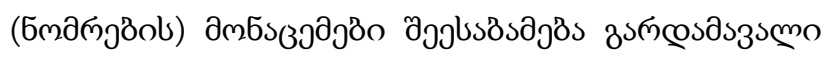

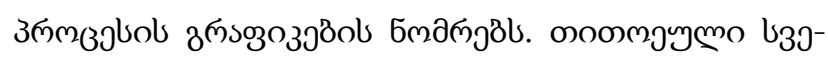

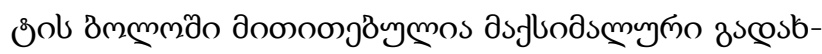

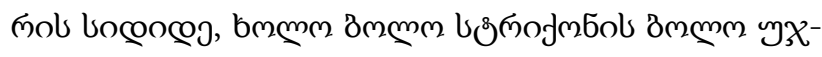

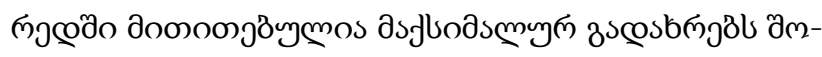

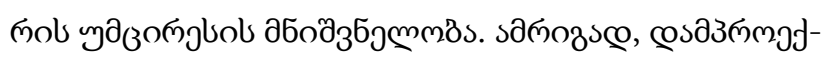

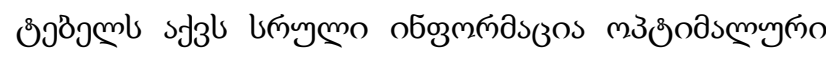

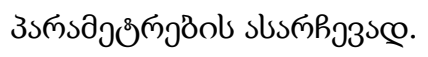

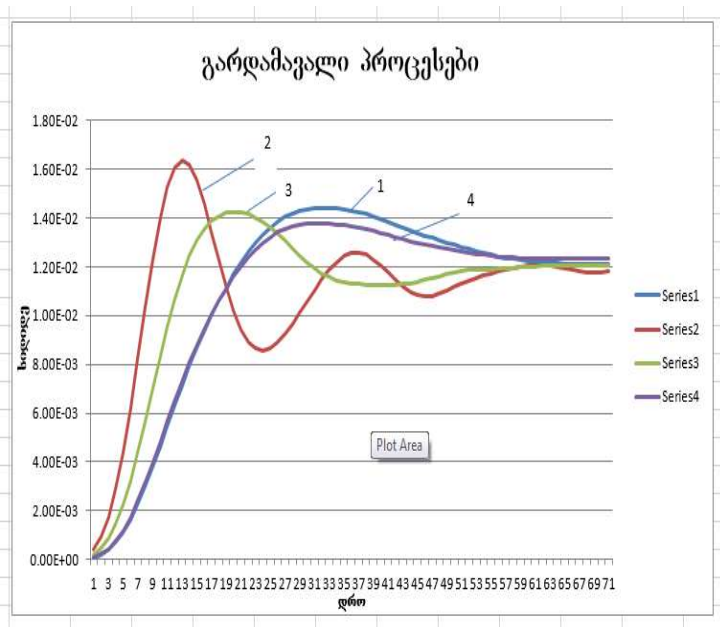

bunm. 3.

\section{cost $33^{6 s}$}

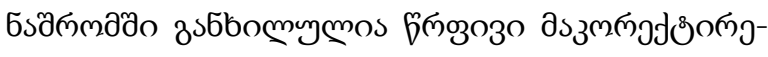

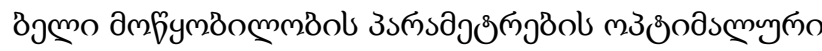

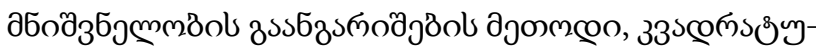

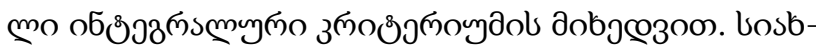

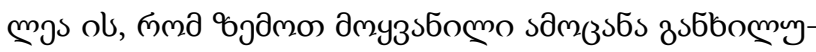

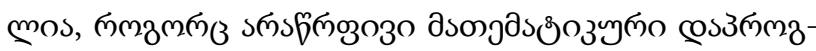

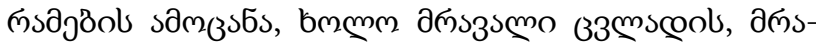
3

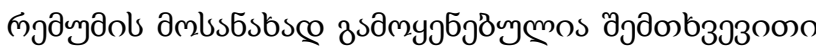

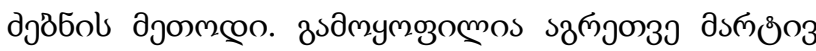

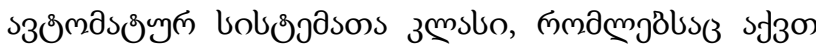

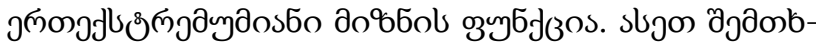
3

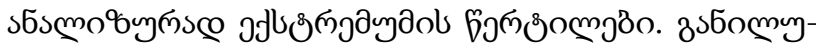

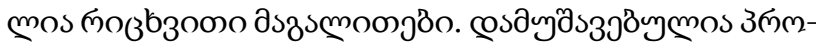

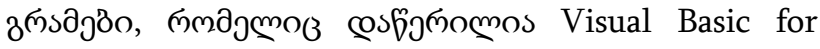

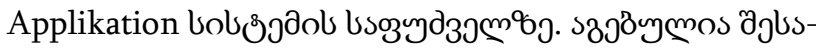

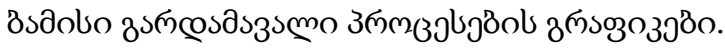




\section{costs ๓ото 1}

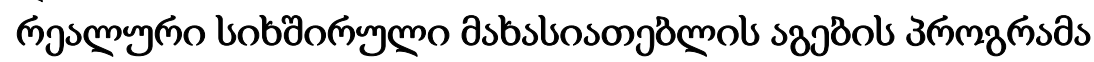

' realuri maxasiatebelis programa

$\operatorname{Dim}$ T1, T2, T3, T4, T01, T02, T03 As Single

Dim c0, c1, c2, c3, c4, c5, D,F, Om, U, q1,q2, k As Single

$\mathrm{T} 1=8.7$

$\mathrm{T} 2=0.47$

$\mathrm{T} 3=200$

$\mathrm{T} 4=0.02$

$\mathrm{T} 01=0.1 \mathrm{~s} \partial \mathrm{s}$

$\mathrm{T} 02=0.02$

$\mathrm{T} 03=0.01$

$\mathrm{k}=200$

$\mathrm{I}=0$

For Om = 0.1 To 50 Step 1

$\mathrm{I}=\mathrm{I}+1$

$\mathrm{cO}=\mathrm{k}$

c1 $=1+\mathrm{k} * \mathrm{~T} 2$

$\mathrm{c} 2=\mathrm{T} 4+\mathrm{T} 1+\mathrm{T} 02+\mathrm{T} 03$

$\mathrm{c} 3=\mathrm{T} 1 * \mathrm{~T} 4+\mathrm{T} 1 * \mathrm{~T} 02+\mathrm{T} 1 * \mathrm{~T} 03+\mathrm{T} 4 * \mathrm{~T} 02+\mathrm{T} 4 * \mathrm{~T} 03+\mathrm{T} 02 * \mathrm{~T} 03$

$\mathrm{c} 4=\mathrm{T} 1 * \mathrm{~T} 4 * \mathrm{~T} 02+\mathrm{T} 1 * \mathrm{~T} 4 * \mathrm{~T} 03+\mathrm{T} 4 * \mathrm{~T} 02 * \mathrm{~T} 03+\mathrm{T} 4 * \mathrm{~T} 02 * \mathrm{~T} 03$

$\mathrm{c} 5=\mathrm{T} 1 * \mathrm{~T} 4 * \mathrm{~T} 02 * \mathrm{~T} 03$

$\mathrm{D}=\mathrm{c} 5 * 0 \mathrm{~m}^{\wedge} 5-\mathrm{c} 3^{*} \mathrm{Om} \mathrm{m}^{\wedge} 3+\mathrm{c} 1^{*} \mathrm{Om}$

$\mathrm{F}=\mathrm{c} 4 * O \mathrm{~m}^{\wedge} 4-\mathrm{c} 2 * O \mathrm{~m}^{\wedge} 2+\mathrm{c} 0$

$\mathrm{U}=\mathrm{c} 0 *(\mathrm{~T} 2 * \mathrm{Om} * \mathrm{D}+\mathrm{f}) /\left(\mathrm{F}^{\wedge} 2+\mathrm{D}^{\wedge} 2\right)$

Cells $(1+\mathrm{I}, 2) \cdot$ Value $=U$

Cells $(1+1,3)$.Value $=$ Om

Next Om

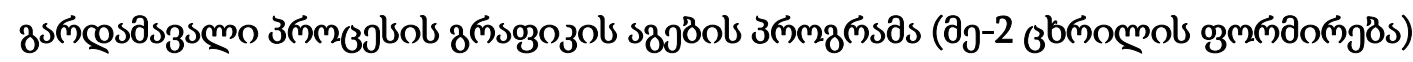

' gardamavalis agebis programa

$\operatorname{Dim}$ T1, T2, T3, T4, T01, T02, T03 As Single

Dim c0, c1, c2, c3, c4, c5, D, F, Om, U, k As Single

Dim $x, y$, delta As Single

Dim I, j As Integer

$\mathrm{T} 1=7.72$

$\mathrm{T} 2=0.325$

$\mathrm{T} 4=0.029$

$\mathrm{k}=214.5$

$\mathrm{T} 01=0.1$

$\mathrm{T} 02=0.02$

$\mathrm{T} 03=0.01$

delta $=0.01$

$y=0$

$\mathrm{I}=0$

For $\mathrm{t}=0.01$ To 0.72 Step 0.01

$\mathrm{I}=\mathrm{I}+1$

For $\mathrm{Om}=0.1$ To 50 Step 1

$\mathrm{cO}=\mathrm{k}$ 


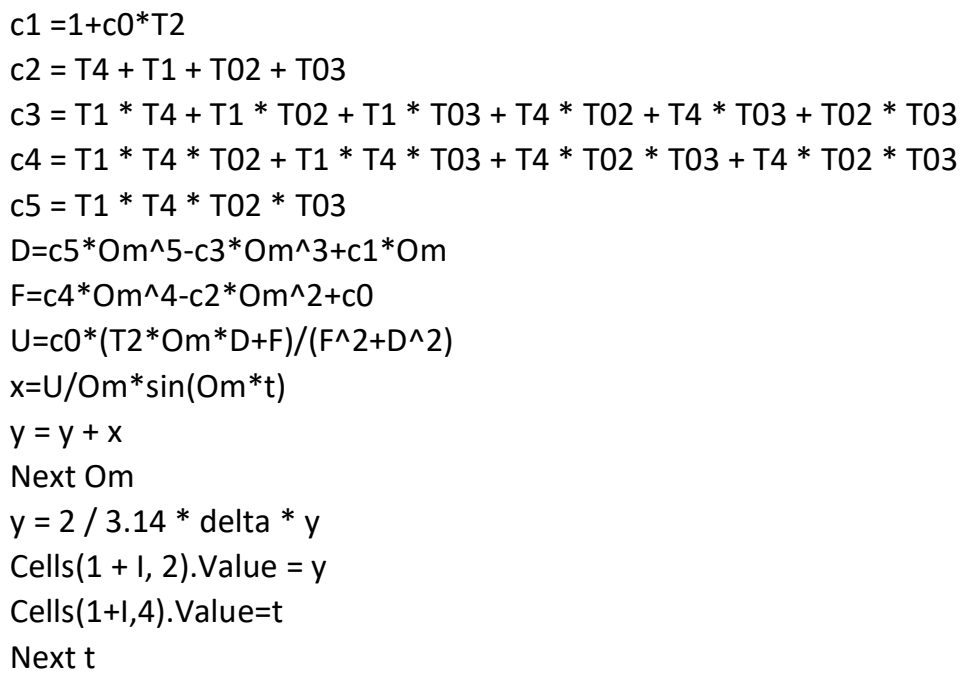




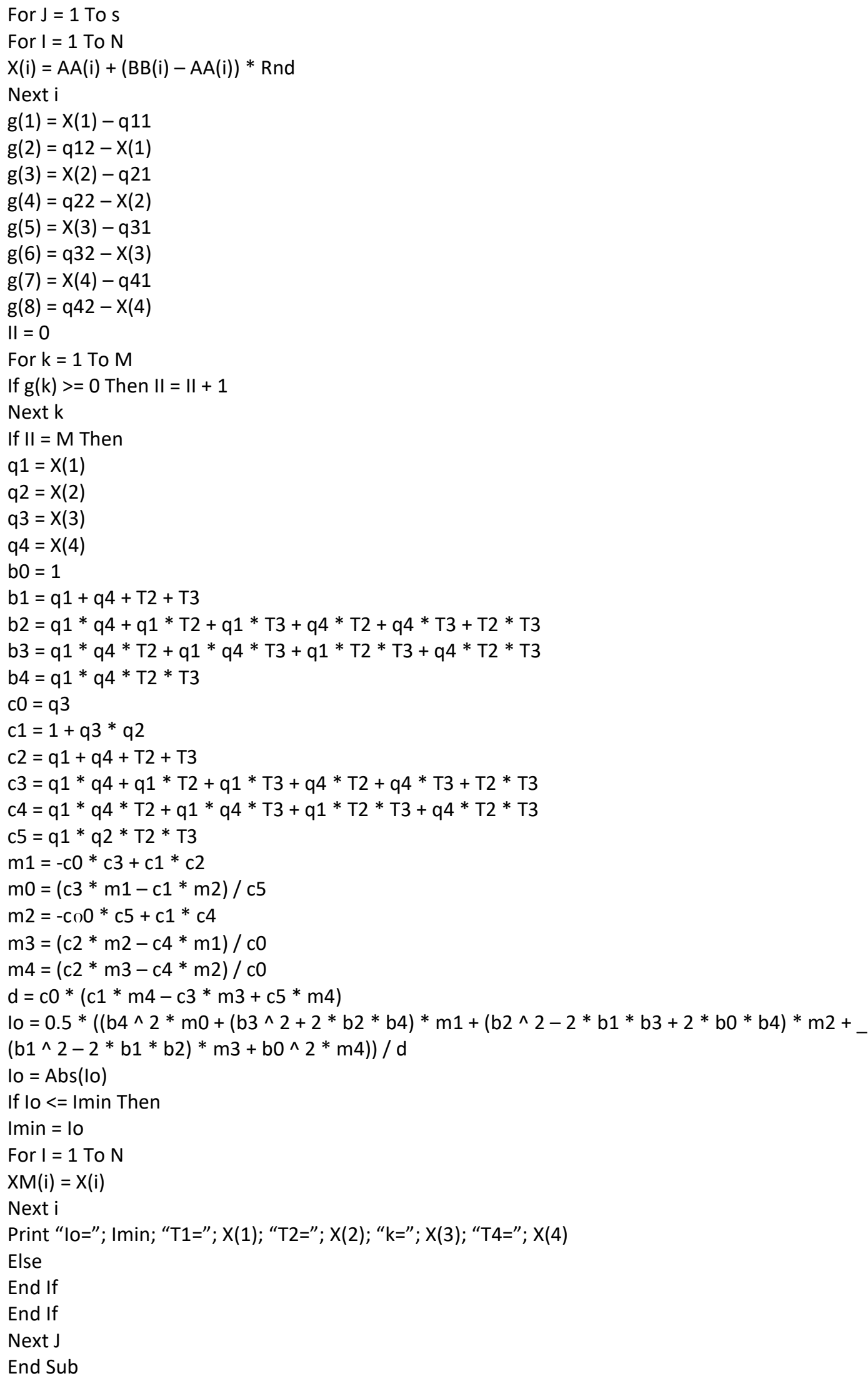




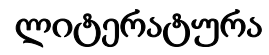

1. V. Lazarev, E. Travina. Synthesis and Calculation of Automatic Control Systems. ITMO University, St. Petersburg, 2018. (In Russian);

2. I. Iboduauli. Solving Problems of Synthesis of Control Systems by the Method of Variational Genetic Programming. Abstract of Dissertation, Moscow: 2014. (In Russian);

3. J.L.K. Newton, L. Gould, J. Kaiser. Theory of Linear Systems (Analytical Methods of Calculation) Fizmat Iz. Moscow: 1961. (In Russian);

4. Theory of Automatic Control. Edited by Netushil. Textbook for universities. Ed. 2nd rev. M.: 1976. (In English);

5. V. Gemin, B. Kagan. Optimal design methods. M.: "Energy", 1980. (In Russian);

6. V. Demidovich, I. Maron. Fundamentals of Computational Mathematics, Moscow: "Science", 1970. (In Russian).

UDC 513.21

SCOPUS CODE 1701

https://doi.org/10.36073/1512-0996-2021-1-55-74

\section{Determination of the Optimal Values of the Parameters of Linear Corrective Devices of the Automatic Control System by the Integral Quadratic Criterion}

Badri Gvasalia

\author{
Department of Computer-aided Design of Construction, Georgian Technical \\ University, Georgia, 0160, Tbilisi, 68 ${ }^{\mathrm{b}} \mathrm{M}$. Kostava str. \\ E-mail: b.gvasalia@gtu.ge
}

\section{Reviewers:}

N. Lominadze, Professor, Faculty of Informatics and Control Systems, GTU

E-mail: n.lominadze@gtu.ge

M. Kublashvili, Professor, Faculty of Construction, GTU

E-mail: m.kublashvili@gtu.ge

Abstract. When designing an automatic control system (ACS), it is important to determine the optimal values of the parameters of the correcting device according to any of the criteria.

Recently, more and more publications have appeared on the use of the method of nonlinear mathematical programming for solving problems of synthesis of an automated control system.

The article discusses a method for determining the optimal parameters of linear correcting devices according to the quadratic integral criterion. The novelty is the presentation of the above mentioned problem in the form of a nonlinear mathematical programming problem. To find a multiparametric, multiextremal, i.e. complex, objective function, a random search method is used.

Also, a class of automatic control systems having one extreme objective function is highlighted. For this case, the theorems are proved, and the formula for determining extreme points is given in an analytical form. Numerical examples are also considered. Programs have been developed for implementing the corresponding algorithms on a computer in VBA language. The graphs of the corresponding transients are given. 
Key words: automatic control system; integral quadratic criteria; linear corrective device; optimal transient process.

UDC 513.21

SCOPUS CODE 1701

https://doi.org/10.36073/1512-0996-2021-1-55-74

\section{Определение оптимальных значений параметров линейных корректирующих устройств системы автоматического управления по интегральному квадратичному критерию}

Бадри Гвасалия Департамент компьютерного проектирования строительства, Грузинский технический университет, Грузия, 0160, Тбилиси ул. М. Костава, $68^{6}$

E-mail: b.gvasalia@gtu.ge

\section{Рецензенты:}

Н. Ломинадзе, профессор факультета информатики и систем управления ГТУ

E-mail: n.lominadze@gtu.ge

М.Кублашвили, профессор строительного факультета ГТУ

E-mail: m.kublashvili@gtu.ge

Аннотация. При проектировании системы автоматического управления (АСУ) важно определить оптимальные значения параметров корректирующего устройства по любому из критериев.

В последнее время появляется все больше публикаций об использовании метода нелинейного математического программирования для решения задач синтеза автоматизированной системы управления.

В статье рассматривается метод определения оптимальных параметров линейных корректирующих устройств по квадратичному интегральному критерию. Новизной является представление выше указанной задачи в виде задачи нелинейного математического программирования. Для нахождения многопараметрической, многоэкстремальной, т.е сложной, целевой функции использован метод случайного поиска.

Также, выделен класс систем автоматического управления, чья целевая функция имеет один экстремум. Для данного случая доказана справедливость теорем, а формула определения экстремальных точек приведена в аналитическом виде. Рассмотрены также численные примеры. Разработаны программы для реализации соответствующих алгоритмов на компьютерном языке VBA. Приведены графики соответствующих переходных процессов.

Ключевые слова: интегральный квадратичный критерии; линейное корректирующее устройство; оптимальный переходный процесс; система автоматического управления.

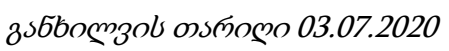

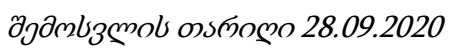

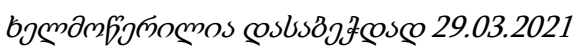

